\title{
Edge-connectivity augmentations of graphs and hypergraphs ${ }^{\star}$
}

\author{
Zoltán Szigeti \\ Laboratoire G-SCOP, INPGrenoble, UJF, CNRS, 46 avenue Félix Viallet 38031 \\ Grenoble Cedex, France
}

Summary. A. Frank (Augmenting graphs to meet edge-connectivity requirements, SIAM J. on Disc. Math. Vol. 5, No. 1 (1992) 22-53) developed a method to solve edge-connectivity augmentation problems. His paper has stimulated further research in a number of directions, including many interesting generalizations.

This paper surveys the current State of the Art on the edge-connectivity augmentation problem. Recent extensions of the problem are presented for undirected graphs, hypergraphs and more generally for set functions. Shortened proofs are provided for some of the results. A list of open problems is also presented.

\subsection{Introduction}

In this paper all graphs and hypergraphs are undirected, directed versions of the problems will not be treated here. By graphs and hypergraphs we mean multi-graphs and multi-hypergraphs, that is, parallel edges and parallel hyperedges are allowed, however loops are forbidden. All the problems here will concern edge-connectivity, that is, vertex-connectivity will not be considered. We have to emphasize immediately that in our problems we may add edges between adjacent vertices, we may even add parallel edges. We remark that without these assumptions, that is, when the starting and also the resulting graph must be simple, the problem is NP-complete [22]. The optimization problems will always be unweighted, the weighted version of the simplest problem already being NP-complete [12].

The basic problem: The starting point for introducing edge-connectivity problems is the problem of increasing the reliability of a telephone network. We may associate a graph to the network: the telephone centers and the connections between them are the vertices and the edges of the graph and the

\footnotetext{
* Some part of this work was done while the author was visiting the Research Institute for Discrete Mathematics, University of Bonn, Lennéstrasse 2, 53113. Bonn, Germany by an Alexander von Humboldt fellowship.
} 
reliability of the network corresponds to the edge-connectivity of this graph. As a natural requirement, we may wish to increase the reliability of the network by constructing new connections between the centers. The optimization problem in the language of graphs is the global edge-connectivity augmentation problem in graphs, namely: Given a graph $G=(V, E)$ and a positive integer $k$, what is the minimum number $\gamma$ of new edges whose addition results in a $k$-edge-connected graph? We show how to solve this problem, in doing so we introduce the key ideas to be applied throughout this paper.

The lower bound: First we provide a lower bound on $\gamma$. Suppose that $G$ is not $k$-edge-connected. This is because there is a set $X$ of degree $d(X)$ less than $k$. Then the deficiency of $X$ is $k-d(X)$, that is, we must add at least $k-d(X)$ edges between $X$ and $V-X$. Let $\left\{X_{1}, \ldots, X_{l}\right\}$ be a subpartition of $V$. The deficiency of this subpartition is the sum of the deficiencies of the $X_{i}$ 's. By adding a new edge we may decrease the deficiency of at most two $X_{i}$ 's so we may decrease the deficiency of the subpartition by at most two, hence we obtain the following lower bound:

$$
\gamma \geq \alpha:=\lceil\text { half of the maximum deficiency of a subpartition of } V\rceil \text {. }
$$

The minimax theorem (see Theorem 3), due to Watanabe and Nakamura [36], saying that this lower bound can always be achieved, can be proved as follows:

Frank's algorithm: (1) Minimal extension: First, add a new vertex $s$ to $G$ and connect it to each vertex of $G$ by $k$ edges. The resulting graph is $k$-edgeconnected in $V$. Secondly, delete as many new edges as possible preserving $k$-edge-connectivity in $V$. The graph obtained is denoted by $G^{\prime}=\left(V, F^{\prime}\right)$. If the degree of $s$ in $G^{\prime}$ is odd, then add an arbitrary new edge incident to $s$. Then we have a graph $G^{\prime \prime}=\left(V+s, E \cup F^{\prime \prime}\right)$ that is $k$-edge-connected in $V$ so that the degree of $s$ is even.

(2) Splitting off: Now we will use the main operation of this paper, called splitting off. Splitting off a pair of edges $s r$, st incident to $s$ means that we delete these two edges and we add a new edge $r t$. Applying Lovász's theorem $2(\mathrm{a})$, split off edge pairs incident to $s$, preserving $k$-edge-connectivity in $V$, as far as the degree of $s$ becomes zero. This way we obtain a $k$-edge-connected graph $G^{*}=(V, E \cup F)$ with $|F|=\frac{\left|F^{\prime \prime}\right|}{2}=\left\lceil\frac{\left|F^{\prime}\right|}{2}\right\rceil$.

Optimality: The optimality will be proved by the existence of a subpartition of $V$ whose deficiency provides equality in (1.1). In $G^{\prime}$, no edge incident to $s$ can be deleted without violating $k$-edge-connectivity in $V$, so each edge $e \in F^{\prime}$ enters a maximal proper subset $X_{e}$ in $V$ of degree $k$, that is, $d_{G}\left(X_{e}\right)+$ $d_{F^{\prime}}\left(X_{e}\right)=k$. By the submodularity of the degree function $d$, these sets provide a subpartition $\left\{X_{1}, \ldots, X_{l}\right\}$ of $V$, for which we do have equality in (1.1):

$$
\gamma \leq|F|=\left\lceil\frac{\left|F^{\prime}\right|}{2}\right\rceil=\left\lceil\frac{1}{2} \sum_{1}^{l} d_{F^{\prime}}\left(X_{i}\right)\right\rceil=\left\lceil\frac{1}{2} \sum_{1}^{l}\left(k-d_{G}\left(X_{i}\right)\right)\right\rceil \leq \alpha \leq \gamma .
$$


Minimal extension: The above method of Frank has two main phases: the first one - minimal extension - consists of the first two steps, while the second one is the splitting off.

The first phase is in fact the construction of a graph $H=(V+s, F)$ with a minimum number of edges such that each edge of $H$ is incident to $s$ and $H$ covers the deficiency function of $G$, that is, for every vertex set $X \subset V$, the number of edges of $H$ leaving $X$ is at least the deficiency of $X$ in $G$ with respect to $k$.

Frank proved in [15] that this can be done not only for the deficiency function of a graph: such an optimal graph $H$ can be constructed that covers a symmetric skew-supermodular function. This result (Theorem 1 in this paper) is not explicitly presented in [15], it was published in [1].

This general result on extension implies that for an edge-connectivity augmentation problem, if the corresponding splitting off exists, then the optimization problem can be solved. Thus we will concentrate on splitting off results in this paper.

Generalizations: The above mentioned basic problem (that is the problem of augmenting global edge-connectivity of a graph by adding graph edges) and its solution capture already the most important ingredients of the theory and they provide a point of departure for studying more complex edge-connectivity augmentation problems. Lots of generalizations of Watanabe and Nakamura's result will be presented here. This paper is divided in three parts: results on graphs, on hypergraphs and on set functions. The results in the different parts are intimately related, we will see that a great number of results on graphs can be generalized for hypergraphs, which in turn can sometimes be further extended to "connectivity" functions.

The first part contains the following generalizations in graphs:

- local edge-connectivity augmentation [15],

- global edge-connectivity augmentation over symmetric parity families [32],

- node to area global edge-connectivity augmentation [19],

- global edge-connectivity augmentation by attaching stars [14],

- local edge-connectivity augmentation by attaching stars [24],

- global edge-connectivity augmentation with partition constraint [2].

We present in the second part generalizations in hypergraphs:

- global edge-connectivity augmentation in hypergraphs by adding graph edges [3],

- global edge-connectivity augmentation in hypergraphs by adding uniform hyperedges [26],

- local edge-connectivity augmentation in hypergraphs by adding graph edges (we mention at once that this problem is NP-complete [11]),

- local edge-connectivity augmentation in hypergraphs by adding a hypergraph of minimum total size [31]. 
The deficiency function with respect to global (resp. local) edge-connectivity in graphs and in hypergraphs is symmetric and crossing supermodular (resp. skew-supermodular). The third part is devoted to generalizations on such set functions:

- covering a symmetric crossing supermodular set function by a graph [6],

- covering a symmetric crossing supermodular set function by a uniform hypergraph [26],

- covering a symmetric skew-supermodular set function by a graph (this problem is NP-complete [27]),

- covering a symmetric semi-monotone set function by a graph [20],

- covering a symmetric skew-supermodular set function by a hypergraph of minimum total size [31].

The main contribution of this paper is to call the reader's attention to Theorem 1 of Frank, to survey the results on the edge-connectivity augmentation problem, to provide short proofs for Theorem 12 on detachments satisfying local edge-connectivity requirements, and for Theorem 14 on partition constrained splitting off preserving global edge-connectivity, and finally, to present some open problems of the theory.

We finish this introduction by emphasizing that we do not attempt to cover all topics of the field, e.g. we do not focus on the design of efficient algorithms, but instead, we concentrate on minimax results of the area.

For further topics, such as Local edge-connectivity augmentation of mixed graphs, Global edge-connectivity augmentation preserving simplicity, Global edge-connectivity augmentation in a graph by adding edges within the members of a partition, Successive edge-connectivity augmentation, Simultaneous global edge-connectivity augmentation, we refer to [1], [4], [5], [9], [23].

\subsection{Definitions}

This section is divided in three parts: definitions on graphs, on hypergraphs and finally on set functions.

Graph: Let $G=(V, E)$ be a graph. Recall that parallel edges are allowed. The set of all subpartitions of $V$ will be denoted by $\mathcal{S}(\boldsymbol{V})$. For a vertex $v \in V, \boldsymbol{\Gamma}(\boldsymbol{v})$ denotes the neighbours of $v$. For $X, Y \subset V, \boldsymbol{G} / \boldsymbol{X}$ denotes the graph obtained from $G$ by contracting $X$ into one vertex, while the resulting parallal edges are kept, $\boldsymbol{d}(\boldsymbol{X}, \boldsymbol{Y})(\operatorname{resp} . \overline{\boldsymbol{d}}(\boldsymbol{X}, \boldsymbol{Y}))$ denotes the number of edges between $X-Y$ and $Y-X($ resp. $X \cap Y$ and $V-(X \cup Y)), \boldsymbol{d}(\boldsymbol{X})=d(X, V-X)$. The set of edges leaving $X$ is called a cut and is denoted by $\boldsymbol{\delta}(\boldsymbol{X})$, that is, $d(X)=|\delta(X)|$. It is well-known and easy to check that, for all $X, Y \subseteq V,(1.2)$ and (1.3) are satisfied. 


$$
\begin{aligned}
& d(X)+d(Y)=d(X \cap Y)+d(X \cup Y)+2 d(X, Y), \\
& d(X)+d(Y)=d(X-Y)+d(Y-X)+2 \bar{d}(X, Y) .
\end{aligned}
$$

The local edge-connectivity between two different vertices $x$ and $y$ of $G$ is defined by $\boldsymbol{\lambda}_{G}(\boldsymbol{x}, \boldsymbol{y})=\min \left\{d_{G}(X): x \in X, y \notin X\right\}$, while $\lambda_{G}(x, x)=+\infty$. By Menger's theorem, $\lambda_{G}(x, y)$ is the maximum number of edge disjoint paths in $G$ between $x$ and $y$. Let $G=(U, E)$ be a graph. For $X \subset U, x, y \in U-X$, $s \in U, u, v \in U-s$ we have

$$
\begin{aligned}
& \lambda_{G / X}(x, y) \geq \lambda_{G}(x, y), \\
& \lambda_{G-s}(u, v) \geq \lambda_{G}(u, v)-\left\lfloor\frac{d_{G}(s)}{2}\right\rfloor .
\end{aligned}
$$

Indeed, if a cut $Q$ separates $x$ and $y$ in $G / X$ then $Q$ also separates them in $G$ and (1.4) follows. On the other hand, if $\mathcal{P}$ is a set of $\lambda_{G}(u, v)$ edge-disjoint paths in $G$ then at most $\left\lfloor\frac{d_{G}(s)}{2}\right\rfloor$ of them may contain the vertex $s$ and hence the other paths of $\mathcal{P}$ belong to $G-s$ and (1.5) follows.

A graph $G=(V, E)$ is called $\boldsymbol{k}$-edge-connected in $\mathbf{U}$ (for some $k \in$ $\mathbb{Z}_{+}$and $\left.U \subseteq V\right)$ if $\lambda_{G}(x, y) \geq k \forall x, y \in U$. This definition will be usually used for $U=V$ or $V-s$ with a specified vertex $s$ of $V$, in which case it is equivalent to (1.6). $\boldsymbol{\lambda}(\boldsymbol{G})$ denotes the global edge-connectivity of $G$ that is the maximum integer $k$ so that $G$ is $k$-edge-connected. Given a symmetric function $r: V \times V \rightarrow \mathbb{Z}_{+}$, we say that $G$ is r-edge-connected if (1.7) is satisfied.

$$
\begin{aligned}
d_{G}(X) & \geq k \quad \forall \emptyset \neq X \subset U, \\
\lambda_{G}(u, v) & \geq r(u, v) \quad \forall u, v \in V .
\end{aligned}
$$

Let $G=(V+s, E)$ be a graph. By splitting off two edges $s r, s t$ we mean the operation that replaces $s r$, st by a new edge $r t$, the new graph will be denoted by $\boldsymbol{G}_{\boldsymbol{r} t}$. Note that we do not allow loops so if $r=t$, then the resulting loop must be deleted. A pair $s r$, st is called $k$-admissible if $G_{r t}$ is $k$-edge-connected in $V$ and it is called $\lambda$-admissible if (1.8) is satisfied. On several occasions, the splitting off will be called $k$-admissible instead of the edge pair. A sequence of splittings off is said to be complete if the degree of $s$ becomes 0 . A complete splitting off is called $k$-admissible ( $\lambda$-admissible) if each splitting off in its sequence is $k$-admissible ( $\lambda$-admissible, respectively).

$$
\lambda_{G_{r t}}(x, y) \geq \lambda_{G}(x, y) \quad \forall x, y \in V .
$$

Hypergraph: Let $\mathcal{G}:=(V, E)$ be a hypergraph, that is $E$ is a multiset of subsets of $V$. The element of $E$ are called hyperedges. Note that parallel hyperedges are allowed. The above definitions of the degree funcion, local edge-connectivity and $k$-edge-connectivity can be naturally generalized for hypergraphs. Indeed, let $\mathcal{G}:=(V, E)$ be a hypergraph. The degree $\boldsymbol{d}_{\mathcal{G}}(\boldsymbol{X})$ 
of a vertex set $X$ is defined as the number of hyperedges intersecting $X$ and $V-X$. The local edge-connectivity between two different vertices $x$ and $y$ of $\mathcal{G}$ is defined by $\boldsymbol{\lambda}_{\mathcal{G}}(\boldsymbol{x}, \boldsymbol{y})=\min \left\{d_{\mathcal{G}}(X): x \in X, y \notin X\right\}$, while $\lambda_{\mathcal{G}}(x, x)=$ $+\infty$. $\mathcal{G}$ is called $\boldsymbol{k}$-edge-connected in $\mathbf{U}$ (for some $k \in \mathbb{Z}_{+}$and $U \subseteq V$ ) if $\lambda_{\mathcal{G}}(x, y) \geq k \forall x, y \in U$. We say that a hypergraph $\mathcal{H}$ covers a function $p$ if

$$
d_{\mathcal{H}}(X) \geq p(X) \quad \forall \emptyset \neq X \subset V .
$$

Call a set $X$ of $V$ tight if $d_{\mathcal{H}}(X)=p(X) . \boldsymbol{c}(\mathcal{G})$ denotes the number of connected components of $\mathcal{G}$. A hypergraph is $\boldsymbol{r}$-uniform if each hyperedge is of size $r$. A graph is a 2-uniform hypergraph. $\mathcal{G}$ is called a 2-3 hypergraph if each hyperedge is of size two or three. The operation $\boldsymbol{\Delta}-\boldsymbol{Y}$ replaces a given 3 -hyperedge $a b c$ by the star $q a, q b, q c$ of a new vertex $q$ of degree three. The operation $\boldsymbol{Y}-\boldsymbol{\Delta}$ replaces the star $q a, q b, q c$ of a given vertex $q$ of degree three by a new 3 -hyperedge $a b c$. It is easy to check that the local edge-connectivities between the original vertices do not change after a $\Delta-Y$ or a $Y-\Delta$ operation.

Set function: Let $p: 2^{V} \rightarrow \mathbb{Z} \cup\{-\infty\}$ be a set function. The function $p$ is called supermodular (crossing supermodular) if (1.10) holds for all $X, Y \subseteq V$ (for all $X, Y \subseteq V$ that are crossing that is $X \cap Y, X-Y, Y-$ $X, V-(X \cup Y) \neq \emptyset)$ and $p$ is called skew-supermodular if at least one of (1.10) and (1.11) hold for all $X, Y \subseteq V$. We say that $p$ is symmetric if (1.12) is satisfied for each $X \subseteq V$. A function $b: 2^{V} \rightarrow \mathbb{Z}$ is called submodular if $-b$ is supermodular. Note that the degree function $d_{G}(X)$ of a graph $G$ is symmetric and, by (1.2), it is submodular.

$$
\begin{aligned}
p(X)+p(Y) & \leq p(X \cap Y)+p(X \cup Y), \\
p(X)+p(Y) & \leq p(X-Y)+p(Y-X), \\
p(X) & =p(V-X) .
\end{aligned}
$$

Given a symmetric function $r: V \times V \rightarrow \mathbb{Z}_{+}$, let us define $\boldsymbol{R}(\boldsymbol{X}):=$ $\max \{r(x, y): x \in X, y \in V-X\}$. It is known that $R$ is skew-supermodular (see e.g. in [16]).

Symmetric crossing supermodular functions generalize the deficiency function of a graph $G$ concerning global edge-connectivity (that is the function $k-d_{G}(X)$ is symmetric crossing supermodular) and symmetric skewsupermodular functions generalize the deficiency function of a graph $G$ concerning local edge-connectivity (that is the function $R(X)-d_{G}(X)$ is symmetric skew-supermodular).

\subsection{Minimal extension}

We start this section with a typical lemma that shows how to use the skewsupermodularity of a function. Recall that if a graph $H$ covers a set function $p$, then a set $X$ of $V$ is called tight if $d_{H}(X)=p(X)$. 
Lemma 1. Let $p: 2^{V} \rightarrow \mathbb{Z} \cup\{-\infty\}$ be a symmetric skew-supermodular set function. Let $H=(V+s, E)$ be a graph that covers $p$. If $X$ and $Y$ are tight sets and $X \cap Y \neq \emptyset$, then either (a) $X \cap Y$ and $X \cup Y$ are tight, or (b) $X-Y$ and $Y-X$ are tight and $\bar{d}(X, Y)=0$.

Proof. We may suppose that $X-Y \neq \emptyset \neq Y-X$ because otherwise (a) is trivially satisfied.

If $p$ satisfies (1.11) for $X$ and $Y$ then, by (1.3) and (1.9),

$$
\begin{aligned}
p(X)+p(Y) & =d_{H}(X)+d_{H}(Y) \\
& =d_{H}(X-Y)+d_{H}(Y-X)+2 \bar{d}(X, Y) \\
& \geq p(X-Y)+p(Y-X)+0 \\
& \geq p(X)+p(Y),
\end{aligned}
$$

so we have equality everywhere, implying (b).

Otherwise, $X \cup Y \neq V$ and $p$ satisfies (1.10) for $X$ and $Y$. Then, by (1.2) and (1.9),

$$
\begin{aligned}
p(X)+p(Y) & =d_{H}(X)+d_{H}(Y) \\
& \geq d_{H}(X \cap Y)+d_{H}(X \cup Y) \\
& \geq p(X \cap Y)+p(X \cup Y) \\
& \geq p(X)+p(Y),
\end{aligned}
$$

so we have equality everywhere, implying (a).

All the augmentation results of this paper will be obtained by applying the following general result of Frank and some suitable splitting off theorem. For the sake of completeness we provide the proof of this theorem.

Theorem 1 (Frank [15], [1]). Let $p: 2^{V} \rightarrow \mathbb{Z} \cup\{-\infty\}$ be a symmetric skew-supermodular function. Then the edgeless graph on $V$ can be extended to a graph $H$ by adding a new vertex $s$ and $\gamma$ edges incident to $s$ so that $H$ covers $p$ if and only if

$$
\sum_{X \in \mathcal{X}} p(X) \leq \gamma \quad \forall \mathcal{X} \in \mathcal{S}(V)
$$

Proof. Suppose that $H=(V+s, E)$ covers $p$, each edge of $H$ is incident to $s$ and $d_{H}(s) \leq \gamma$. Then, for any subpartition $\mathcal{X}$ of $V,(1.13)$ is satisfied by

$$
\sum_{X \in \mathcal{X}} p(X) \leq \sum_{X \in \mathcal{X}} d_{H}(X) \leq d_{H}(s) \leq \gamma .
$$

Now suppose that (1.13) is satisfied. The desired graph is constructed as follows. First add a new vertex $s$ to $V$ and connect it to each vertex of $V$ by $\max \{p(X): X \subset V\}$ edges. Then, of course, this graph covers $p$. Secondly, 
delete as many edges as possible preserving that $p$ is covered. Let $H$ be the graph obtained. It remains to show that $d_{H}(s) \leq \gamma$.

No edge of $H$ can be deleted, that is, each edge of $H$ enters a tight set. Thus there exists a set $\mathcal{X}:=\left\{X_{1}, \ldots, X_{l}\right\}$ of tight sets so that each edge enters some set $X_{i}$ and $\sum_{1}^{l}\left|X_{i}\right|$ is minimal.

We claim that $\mathcal{X}$ is a subpartition of $V$. Suppose that $X_{i} \cap X_{j} \neq \emptyset$ for some $X_{i}, X_{j} \in \mathcal{X}$. By Lemma 1 , either $X_{i} \cup X_{j}$ is tight, hence $X_{i}$ and $X_{j}$ can be replaced by $X_{i} \cup X_{j}$ or $X_{i}-X_{j}$ and $X_{j}-X_{i}$ are tight and $\bar{d}\left(X_{i}, X_{j}\right)=0$ (that is no edge enters $X_{i} \cap X_{j}$ ), so $X_{i}$ and $X_{j}$ can be replaced by $X_{i}-X_{j}$ and $X_{j}-X_{i}$. In both cases we obtained a contradiction to the fact that $\sum_{1}^{l}\left|X_{i}\right|$ is minimal.

Thus, $\mathcal{X} \in \mathcal{S}(V)$, so we are done because, by (1.13),

$$
d_{H}(s)=\sum_{X \in \mathcal{X}} d_{H}(X)=\sum_{X \in \mathcal{X}} p(X) \leq \gamma .
$$

\subsection{Graphs}

In this section we will present the problems on edge-connectivity augmentation in graphs and their solutions. As we mentioned in the introduction, the basic tool is splitting off. Each subsection is devoted to one problem, and it is divided in two parts : results on splitting off and then, applying Theorem 1, we read out the minimax result on augmentation.

\subsubsection{Global edge-connectivity I}

This section is about $k$-edge-connectivity for some $k \geq 2$. First we consider the operation splitting off: here the graph $G=(V+s, E)$ is $k$-edge-connected in $V$ and we wish to reduce the graph (by splitting off an edge pair incident to $s$ ) in such a way that the graph remains $k$-edge-connected in $V$. Then we consider the augmentation problem where the aim is to make a graph $k$-edge-connected by adding new edges.

\section{Splitting off preserving global edge-connectivity}

The first result on splitting off is due to Lovász [28]. It concerns global edgeconnectivity, namely it provides a sufficient condition for the existence of a $k$-admissible splitting off. More precisely, Lovász showed that if $k \geq 2$ and the degree of the special vertex $s$ is even, then each edge belongs to a $k$-admissible pair at $s$. In [2] it was shown that each edge belongs to many $k$-admissible pairs.

Theorem 2. Let $G=(V+s, E)$ be a k-edge-connected graph in $V$ with $k \geq 2$ and $d(s)$ is even. Then: 
(a) (Lovász [28]) each edge st belongs to a $k$-admissible pair at $s$.

(b) (Bang-Jensen, Gabow, Jordán, Szigeti [2]) each edge st belongs to at least $\frac{d(s)}{2}$ (resp. $\left.\frac{d(s)}{2}-1\right) k$-admissibles pairs at $s$ if $k$ is even (resp. odd).

B. Fleiner [14], and independently Bang-Jensen and Jackson [3] proved that Theorem 2(a) is true for 2-3 hypergraphs containing no 3-hyperedges incident to $s$. The special case of Theorem 2(b), when $G$ is Eulerian, was proved earlier by Jackson [21].

We must emphasize that the above theorem is true only if $k \geq 2$. Let us consider the following example for $k=1$ : let the graph $G=(V+s, E)$ be the star of the vertex $s$ of degree four. Then $G$ is connected in $V$ and there is no complete 1-admissible splitting off. To avoid this problem we will usually suppose that the connectivity requirement is at least two.

\section{Augmentation of global edge-connectivity by adding edges}

The problem of global edge-connectivity augmentation in graphs, already introduced and also solved in the introduction, is the following: Given a graph $G=(V, E)$ and an integer $k$, what is the minimum number $\gamma$ of new edges whose addition results in a $k$-edge-connected graph? In other words, we are looking for

$$
\begin{aligned}
\gamma & :=\min \left\{\left|E^{\prime}\right|: d_{G+E^{\prime}}(X) \geq k \forall \emptyset \neq X \subset V\right\} \\
& =\min \left\{\left|E^{\prime}\right|: d_{\left(V, E^{\prime}\right)}(X) \geq k-d_{G}(X) \forall \emptyset \neq X \subset V\right\} .
\end{aligned}
$$

As the function $p(X)=k-d_{G}(X)$ is symmetric and, by (1.2), skewsupermodular, Theorems 1 and 2(a) imply the following theorem. (Cai and Sun [8] also proved this result later using some splitting off technique.)

Theorem 3 (Watanabe and Nakamura [36]). Let $G=(V, E)$ be a graph and $k \geq 2$. Then $G$ can be made $k$-edge-connected by adding at most $\gamma$ new edges if and only if

$$
\sum_{X \in \mathcal{X}}\left(k-d_{G}(X)\right) \leq 2 \gamma \quad \forall \mathcal{X} \in \mathcal{S}(V) .
$$

We point out again that the case when $k=1$ does not fit into this framework. It is obvious that in this case we have to add $l-1$ new edges, where $l$ is the number of connected components of the graph.

\subsubsection{Local edge-connectivity I}

In the above section we were interested in $k$-edge-connectivity, that is, in the minimum value of the local edge-connectivities over all pairs of vertices. This section concerns the problem where each value counts not just the minimum, that is, we wish to add new edges to a graph so that the local edge-connectivity be greater than or equal to a given requirement for each pair of vertices. 


\section{Splitting off preserving local edge-connectivity}

In this section we summarize results on splitting off preserving local edgeconnectivity. Mader [29] generalized Lovász's result Theorem 2(a) for local edge-connectivity by showing that a $\lambda$-admissible pair always exists if the degree of the vertex $s$ is different from 3 and roughly speaking $G$ is 2-edgeconnected. This result implies that at most three edges incident to $s$ belong to no $\lambda$-admissible pair. Frank [16] improved this by showing that at most one edge incident to $s$ belongs to no $\lambda$-admissible pair. In [33] we characterized this edge. Since not every edge belongs to a $\lambda$-admissible pair, the best we may hope for is that there exists at least one edge that belongs to many $\lambda$ admissible pairs. In [33] it is shown that the correct number is $\left\lfloor\frac{d(s)}{3}\right\rfloor$ and examples show that this result is best possible.

Theorem 4. Let $G=(V+s, E)$ be a connected graph so that $d(s) \neq 3$ and no cut edge is incident to $s$.

(a) (Mader [29]) There exists a $\lambda$-admissible pair at $s$.

(b) (Frank [16]) There exist $\left\lfloor\frac{d(s)}{2}\right\rfloor$ disjoint $\lambda$-admissible pairs at s. (Hence at most one edge incident to $s$ belongs to no $\lambda$-admissible pair.)

(c) (Szigeti [33]) An edge st belongs to no $\lambda$-admissible pair if and only if $d(s)$ is odd and there exist two disjoint sets $C_{1}, C_{2} \subset V-t$ such that $d\left(C_{i}\right)=R\left(C_{i}\right)$ and $d\left(s, C_{i}\right)=\frac{d(s)-1}{2}$ for $i=1,2$. Moreover, for every $c_{1} \in C_{1} \cap \Gamma(s), c_{2} \in C_{2} \cap \Gamma(s),\left\{s c_{1}, s c_{2}\right\}$ is a $\lambda$-admissible pair.

(d) (Szigeti [33]) There exists an edge belonging to at least $\left\lfloor\frac{d(s)}{3}\right\rfloor \lambda$-admissible pairs at $s$.

\section{Augmentation of local edge-connectivity by adding edges}

The problem of local edge-connectivity augmentation in graphs is defined as follows: Given a graph $G$ and a symmetric requirement function $r: V \times V \rightarrow \mathbb{Z}_{+}$, what is the minimum number $\gamma$ of new edges whose addition results in an $\mathbf{r}$-edge-connected graph?

Note that, by taking $r$ to be equal to $k$ for each pair of vertices, this problem contains, as a special case, the global edge-connectivity augmentation problem in graphs.

Recall that $R(X)=\max \{r(x, y): x \in X, y \in V-X\}$ is symmetric and skew-supermodular. We can reformulate the problem as follows: we look for

$$
\begin{aligned}
\gamma & :=\min \left\{\left|E^{\prime}\right|: \lambda_{G+E^{\prime}}(u, v) \geq r(u, v) \forall u, v \in V\right\} \\
& =\min \left\{\left|E^{\prime}\right|: d_{G+E^{\prime}}(X) \geq R(X) \forall \emptyset \neq X \subset V\right\} \\
& =\min \left\{\left|E^{\prime}\right|: d_{\left(V, E^{\prime}\right)}(X) \geq R(X)-d_{G}(X) \forall \emptyset \neq X \subset V\right\} .
\end{aligned}
$$

As $p(X)=R(X)-d_{G}(X)$ is a symmetric, skew-supermodular function, Theorems 1 and 4 imply the following theorem. 
Theorem 5 (Frank [15]). Let $G=(V, E)$ be a graph and $2 \leq r(u, v) \in$ $\mathbb{Z} \forall u, v \in V$. Then $G$ can be made $\mathbf{r}$-edge-connected by adding at most $\gamma$ new edges if and only if

$$
\sum_{X \in \mathcal{X}}\left(R(X)-d_{G}(X)\right) \leq 2 \gamma \quad \forall \mathcal{X} \in \mathcal{S}(V) .
$$

Note that the special case of Theorem 5 when $r(u, v)=k \forall u, v \in V$ is exactly Theorem 3 .

\subsubsection{Symmetric parity families}

A family $\mathcal{F}$ of subsets of $V$ is called a symmetric parity family if it satisfies the following three properties. (i) $\emptyset, V \notin \mathcal{F}$, (ii) if $A \in \mathcal{F}$, then $V-A \in \mathcal{F}$, (iii) if $A, B \notin \mathcal{F}$ and $A \cap B=\emptyset$, then $A \cup B \notin \mathcal{F}$. Let $T \subseteq V$ be a set of even cardinality. A set $X$ is called T-odd if $|T \cap X|$ is odd. If $G=(V, E)$ is a connected graph then $(G, T)$ is called a graft. A cut $\delta_{G}(X)$ is called a T-cut if $X$ is T-odd, more generally $\delta_{G}(X)$ is called an $\mathcal{F}$-cut if $X \in \mathcal{F}$. The most important examples of parity families are $\mathcal{F}:=2^{V}-\{\emptyset, V\}$ and $\mathcal{F}:=\{X \subseteq V: X$ is T-odd $\}$.

In this section we will deal with $\mathcal{F}$-cuts.

\section{Splitting off preserving global edge-connectivity over symmetric} parity families

Theorem 4(a) implies easily the following.

Theorem 6 (Szigeti [32]). Let $G=(V+s, E)$ be a graph so that $d(s)>0$ is even and let $\mathcal{F}$ be a symmetric parity family on $V$. Suppose that for some $k \geq 2$,

$$
d(X) \geq k \quad \forall X \in \mathcal{F} .
$$

Then there exists a pair of edges incident to $s$ that can be split off without violating (1.16).

\section{Augmentation of global edge-connectivity over symmetric parity families}

In this section we solve the following global edge-connectivity augmentation problem over a symmetric parity family: Given a graph $G=(V, E)$, a symmetric parity family $\mathcal{F}$ on $V$ and an integer $k$, what is the minimum number $\gamma$ of edges whose addition results in a graph in which each $\mathcal{F}$-cut contains at least $k$ edges?

A special case is the minimum T-cut augmentation problem: how many new edges must be added to a graph so that the minimum T-cut contains at 
least $k$ edges? It also contains, as a special case, the global edge-connectivity augmentation problem in graphs.

For a symmetric parity family $\mathcal{F}$, a graph $G=(V, E)$ and $k \in \mathbb{Z}^{+}$, let $p(X):=k-d_{G}(X)$ if $X \in \mathcal{F}$, and $-\infty$ otherwise. The problem of edgeconnectivity augmentation over a symmetric parity family can be reformulated as follows: what is the value

$$
\begin{aligned}
\gamma & :=\min \left\{\left|E^{\prime}\right|: d_{G+E^{\prime}}(X) \geq k \forall X \in \mathcal{F}\right\} \\
& =\min \left\{\left|E^{\prime}\right|: d_{\left(V, E^{\prime}\right)}(X) \geq p(X) \forall X \subseteq V\right\} ?
\end{aligned}
$$

It is easy to see that $p$ is a symmetric skew-supermodular function, so Theorems 1 and 6 provide at once the following theorem.

Theorem 7 (Szigeti [32]). For a graph $G=(V, E)$, a symmetric parity family $\mathcal{F}$ on $V$ and an integer $k \geq 2$, the minimum cardinality of an $\mathcal{F}$-cut can be augmented to $k$ by adding at most $\gamma$ edges if and only if

$$
\sum_{i=1}^{l}\left(k-d\left(X_{i}\right)\right) \leq 2 \gamma \quad \forall\left\{X_{1}, \ldots, X_{l}\right\} \in \mathcal{S}(V) \text { with } X_{i} \in \mathcal{F}
$$

By applying Theorem 7 , for $\mathcal{F}=2^{V}-\{\emptyset, V\}$ we get Theorem 3 , and, for $\mathcal{F}$ being the set of T-odd subsets of $V$, we get the following theorem on T-cuts.

Theorem 8 (Szigeti [32]). For any graft $(G, T)$, the minimum cardinality of a T-cut can be augmented to $k \geq 2$ by adding at most $\gamma$ edges if and only if $\sum_{X \in \mathcal{X}}(k-d(X)) \leq 2 \gamma$ for each subpartition $\mathcal{X}$ of $V$ into $T$-odd sets.

\subsubsection{Node to area edge-connectivity}

The node to area global edge-connectivity augmentation problem can be defined as follows: Given a graph $G=(V, E)$, a family $\mathcal{A}$ of sets $A \subseteq V$ (called areas), and a requirement function $r: \mathcal{A} \rightarrow \mathbb{Z}_{+}$, add a minimum number $\boldsymbol{O p t}(\boldsymbol{r}, \boldsymbol{G})$ of new edges to $G$ so that the resulting graph contains $r(A)$ edge-disjoint paths from any area $A$ to any vertex $v \notin A$.

Note that, by taking just one vertex as the family of areas and $k$ as the requirement for this vertex, then we get as a special case the global edgeconnectivity augmentation problem in graphs.

Let us define $P_{\mathcal{A}}(X)=\max \{r(A): A \in \mathcal{A}, A \cap X=\emptyset$ or $A \subseteq X\}$ if $V \neq X \neq \emptyset$ and $P_{\mathcal{A}}(V)=P_{\mathcal{A}}(\emptyset)=0$ and $Q_{\mathcal{A}}(G):=\max \left\{\sum_{X \in \mathcal{X}} q_{\mathcal{A}}(X): \mathcal{X} \in\right.$ $\mathcal{S}(V)\}$, where $q_{\mathcal{A}}(X)=P_{\mathcal{A}}(X)-d_{G}(X)$.

Now we can provide a lower bound for the optimal value, namely $\operatorname{Opt}(r, G)$ $\geq\left\lceil\frac{Q_{\mathcal{A}}(G)}{2}\right\rceil$. The question is whether we have always equality here or not. Usually equality will hold, unless the graph contains a special configuration. 
The node to area global edge-connectivity augmentation problem is a special case of the symmetric semi-monotone function covering problem treated in Section 1.6.2, so Theorem 29 implies the following. We mention that the above defined $P_{\mathcal{A}}(X)$ is the symmetric semi-monotone function to be covered.

Theorem 9 (Ishii, Hagiwara [19]). Let $G=(V, E)$ be a graph, $\mathcal{A}$ a family of sets $A \subseteq V$, and $r: \mathcal{A} \rightarrow \mathbb{Z}_{+}$a requirement function so that $r(A) \neq 1 \forall A \in$ $\mathcal{A}$. If $G$ contains no $\mathcal{A}$-configuration, then $\operatorname{Opt}(r, G)=\left\lceil\frac{Q_{\mathcal{A}}(G)}{2}\right\rceil$, otherwise $\operatorname{Opt}(r, G)=\left\lceil\frac{Q_{\mathcal{A}}(G)}{2}\right\rceil+1$.

The definition of a $\mathcal{A}$-configuration can be found in [19], where it is called P-property. We mention that if $\mathcal{A}=\{v\}$ and $r(v)=k$, then no $\mathcal{A}$-configuration can exist, so Theorem 9 implies Theorem 3. We remark that without the condition $r(A) \neq 1 \forall A \in \mathcal{A}$, the problem is NP-complete, see Section 1.6.2.

\subsubsection{Global edge-connectivity II}

Let us return to global edge-connectivity. First we generalize the operation splitting off, by introducing detachment, and then we consider the problem where we wish again to make a graph $k$-edge-connected, but this time by attaching stars. The essential tool to solve this problem is exactly the operation detachment.

\section{Detachments preserving global edge-connectivity}

In this section we generalize the operation splitting off.

Let $G=(V+s, E)$ be a graph. A degree specification for $s$ is a sequence $\boldsymbol{f}(s)=\left(d_{1}, \ldots, d_{p}\right)$ of positive integers with $\sum_{j=1}^{p} d_{j}=d_{G}(s)$. An $f(s)$-detachment of $G$ at $s$ is the graph $G^{\prime}$ obtained from $G$ by replacing $s$ by a set $s_{1}, \ldots, s_{p}$ of independent vertices and distributing the edges incident to $s$ among them in such a way that $d_{G^{\prime}}\left(s_{i}\right)=d_{i}(1 \leq i \leq p)$. Note that all the other ends of the edges in $G$ remain the same.

Let us mention that a splitting off can really be considered as a special case of detachment, namely if after a splitting off we subdivide the new edge then the new graph is a $(2, d(s)-2)$-detachment and this operation does not change the local edge-connectivities between the original vertices.

The following beautiful theorem of B. Fleiner characterizes graphs that have a $k$-edge-connected $f(s)$-detachment.

Theorem 10 (B.Fleiner [14]). Let $G=(V+s, E)$ be a graph, $2 \leq k \in \mathbb{Z}$ and $f(s)=\left(d_{1}, \ldots, d_{p}\right)$ a degree specification for $s$ with $d_{i} \geq 2 \forall i$. Then there exists an $f(s)$-detachment of $G$ that is $k$-edge-connected in $V$ if and only if

$$
\begin{gathered}
G \text { is } k \text {-edge-connected in } V, \\
G-s \text { is }\left(k-\sum_{1}^{p}\left\lfloor\frac{d_{i}}{2}\right\rfloor\right) \text {-edge-connected. }
\end{gathered}
$$


We note that the special case of Theorem 10, when each $d_{i}$ is even, is equivalent to Theorem 2(a). Indeed, in this case the condition (1.19) is automatically satisfied by (1.5) and hence Theorem 10 implies Theorem 2(a). On the other hand, by Theorem $2(\mathrm{a})$, there exists a complete $k$-admissible splitting off, subdividing each new edge by a vertex and combining the suitable number of new vertices, an $f(s)$-detachment is obtained which is $k$-edge-connected in $V$ by (1.4), and hence Theorem 2(a) implies this special case of Theorem 10.

The proof technique of B. Fleiner [14] needed the more general framework of 2-3 hypegraphs. He proved that Theorem 10 is true for 2-3 hypergraphs containing no 3-hyperedges incident to $s$.

We give a generalization of this result in Section 1.4.6, for which we will provide a short proof in the appendix, much shorter than the original proof of B. Fleiner of Theorem 10.

\section{Augmentation of global edge-connectivity by attaching stars}

By attaching a star of degree $d$ to a graph $G$, we mean adding a new vertex and connecting it to some vertices of $G$ so that the degree of the new vertex becomes $d$. The problem of this section is the global edge-connectivity augmentation problem of a graph by attaching stars that can be defined as follows: Given a graph $G=(V, E)$ and integers $k, d_{1}, \ldots, d_{p}$, decide whether it is possible to attach $p$ stars to $G$ with degrees $d_{1}, \ldots, d_{p}$ to have a $k$-edge-connected graph in $V$.

Note that, by taking each $d_{i}$ to be equal to 2 , we get as a special case the global edge-connectivity augmentation problem in a graph.

The solution of the problem of this section is given in the following theorem of B. Fleiner which is implied by Theorems 1 and 10. It might be advantageous to notice that condition (1.20) and the fact that $k-d(X)$ is symmetric skewsupermodular guarantees that the minimal extension can be made by $\sum_{j=1}^{p} d_{j}$ edges, while condition (1.21) allows us to find the suitable detachment.

Theorem 11 (B. Fleiner [14]). A graph $G=(V, E)$ can be made $k$-edgeconnected $(k \geq 2)$ by attaching $p$ stars with degrees $d_{1}, \ldots, d_{p}\left(d_{i} \geq 2 \forall i\right)$ if and only if

$$
\begin{aligned}
& \sum_{X \in \mathcal{X}}(k-d(X)) \leq \sum_{j=1}^{p} d_{j} \quad \forall \mathcal{X} \in \mathcal{S}(V), \\
& k-\sum_{j=1}^{p}\left\lfloor\frac{d_{j}}{2}\right\rfloor \leq \lambda(G) .
\end{aligned}
$$

Note that, for $d_{i}=2 \forall i,(1.21)$ is satisfied by (1.20). Indeed, for $X \subset V$ with $d(X)=\lambda(X)$, by $(1.20)$ for $\{X, V-X\},(k-d(X))+(k-d(V-X)) \leq 2 p$, so by the symmetry of $d(X), k-p \leq d(X)=\lambda(G)$, that is, (1.21) is satisfied. Hence Theorem 11 implies Theorem 3. 


\subsubsection{Local edge-connectivity II}

In this section we generalize the results of the previous section. First we extend the Theorem of B. Fleiner to the case when local edge-connectivity is involved, and then we solve the problem where we wish to attach stars of given degree to a graph so that the local edge-connectivity be greater than or equal to a given requirement for each pair of vertices.

\section{Detachments preserving local edge-connectivity}

Recall that $G=(V+s, E)$ is r-edge-connected if $\lambda_{G}(u, v) \geq r(u, v) \forall u, v \in V$, where $r: V \times V \rightarrow \mathbb{Z}_{+}$is a symmetric requirement function. In the next result we characterize graphs that have an $\mathbf{r}$-edge-connected $f(s)$-detachment.

Note that, by taking $\mathbf{r}$ to be equal to $k$ for each pair of vertices, we get a characterization of graphs having a $k$-edge-connected $f(s)$-detachment.

Theorem 12 (Jordán, Szigeti [24]). Let $G=(V+s, E)$ be a graph, $\boldsymbol{r}$ a symmetric requirement function on $V$ with $r(u, v) \geq 2 \forall u, v \in V$ and $f(s)=$ $\left(d_{1}, \ldots, d_{p}\right)$ a degree specification for $s$ with $d_{i} \geq 2 \forall i$. Let $\varphi=\sum_{1}^{p}\left\lfloor\frac{d_{i}}{2}\right\rfloor$. Then there exists an $\mathbf{r}$-edge-connected $f(s)$-detachment of $G$ if and only if

$$
\begin{aligned}
G & \text { is } \mathbf{r} \text {-edge-connected, } \\
G-s & \text { is }(\mathbf{r}-\boldsymbol{\varphi}) \text {-edge-connected. }
\end{aligned}
$$

The special case of Theorem 12, when $r(u, v)=k \forall u, v \in V$, is Theorem 10 , and when $r(u, v)=\lambda_{G}(u, v) \forall u, v \in V$, provides a characterization of the existence of an $f(s)$-detachment that preserves local edge-connectivities, while when at most one $d_{i}$ is odd, is equivalent to Theorem 4(a).

We will provide a short proof of the above theorem in Section 1.8.1. It will use Theorem 4(a) so it does not provide a new proof for Theorem 4(a). Note that it provides a short proof for Theorem 10 .

\section{Augmentation of local edge-connectivity by attaching stars}

In this section we solve the following global edge-connectivity augmentation problem of a graph by attaching stars: Given a graph $G=(V, E)$, a symmetric requirement function $r: V \times V \rightarrow \mathbb{Z}_{+}$and integers $d_{1}, \ldots, d_{p}$, decide whether it is possible to attach $p$ stars to $G$ with degrees $d_{1}, \ldots, d_{p}$, so as to obtain an $\mathbf{r}$-edge-connected graph in $V$.

Note that, by taking $\mathbf{r}$ to be equal to $k$ for each pair of vertices, we get as a special case the global edge-connectivity augmentation problem of a graph by attaching stars, and by taking each $d_{i}$ to be equal to 2 but $\mathbf{r}$ being arbitrary, we get the local edge-connectivity augmentation problem. 
Recall that $R(X)=\max \{r(x, y): x \in X, y \in V-X\}$ and that the function $R(X)-d(X)$ is symmetric skew-supermodular. Then Theorems 1 and 12 imply at once the following theorem. As for the global case, we may notice that condition (1.24) guarantees that the minimal extension can be made by $\sum_{j=1}^{p} d_{j}$ edges, while condition (1.25) allows us to find the suitable detachment.

Theorem 13 (Jordán, Szigeti [24]). Let $G=(V, E)$ be a graph, $r: V \times$ $V \rightarrow \mathbb{Z}_{+}$a symmetric requirement function with $r(u, v) \geq 2 \forall u, v \in V$. Then $G$ can be made $\mathbf{r}$-edge-connected by attaching $p$ stars with degrees $d_{1}, \ldots, d_{p}\left(d_{i} \geq\right.$ $2 \forall i$ ) if and only if

$$
\begin{array}{r}
\sum_{X \in \mathcal{X}}(R(X)-d(X)) \leq \sum_{j=1}^{p} d_{j} \quad \forall \mathcal{X} \in \mathcal{S}(V), \\
r(u, v)-\sum_{j=1}^{p}\left\lfloor\frac{d_{j}}{2}\right\rfloor \leq \lambda_{G}(u, v) \quad \forall u, v \in V .
\end{array}
$$

Note that, if $r(u, v)=k \forall u, v \in V$, then (1.24) and (1.25) are equivalent to (1.20) and (1.21), so Theorem 13 implies Theorem 11, and if $d_{i}=2 \forall i$, then (1.24) implies (1.25) (that can be shown similarly, as it was shown for the global case) and hence Theorem 13 implies Theorem 5. Hence, our result is a common generalization of B. Fleiner's theorem on global edge-connectivity aumentation by attaching stars and Frank's theorem on local edge-connectivity aumentation by adding edges.

\subsubsection{Globel edge-connectivity with partition constraints}

The aim of this section is to present the solution of the problem of global edge-connectivity augmentation in bipartite graphs. In fact, we consider a more general setting, namely the global edge-connectivity augmentation problem of a graph with partition constraints: we want to make an arbitrary graph $k$-edge-connected by adding a minimum number of new edges between different members of a given partition of the vertex set.

\section{Splitting off preserving global edge-connectivity with partition constraints}

Let $G=(V+s, E)$ be a graph with a specified vertex $s$ of even degree, and $\mathcal{P}=\left\{P_{1}, \ldots, P_{r}\right\}$ a partition of $V($ resp. $\delta(s))$. If we have a partition of $V$, then obviously we may define a partition of $\delta(s)$, hence the second form is more general than the first one. A splitting off $\{s u, s v\}$ is called $\mathcal{P}$-allowed if $u$ and $v$ (resp. $s u$ and $s v$ ) belong to different members of $\mathcal{P}$. A $k$-admissible $\mathcal{P}$-allowed splitting off will be called allowed. We wish to characterize graphs and partitions for which there exists a complete allowed splitting off, that 
is, we are interested in a complete splitting off, that is, at the same time $\mathcal{P}$ allowed and $k$-admissible. Notice that a complete $\mathcal{P}$-allowed splitting off exists if and only if $d\left(s, P_{i}\right) \leq \frac{d(s)}{2} \forall 1 \leq i \leq r$, while, as it is already known for us, a complete $k$-admissible splitting off exists if and only if $G$ is $k$-edge-connected in $V$. Are these conditions together sufficient to have an allowed complete splitting off? We will answer this question in this section.

To show the difficulties of our problem, suppose we wish to make 3-edgeconnected a 4 -cycle $C_{4}$. Clearly, this can be done by adding two edges. Note that the optimal solution is unique, we have to transform the graph into a $K_{4}$. Now suppose that we have an additional condition, we must maintain the bipartiteness of $C_{4}$. Then this solution is not feasible any more. In this case we have to add 3 edges. Let us reformulate this difficulty in terms of splitting off. Let $G$ be the graph obtained from $C_{4}$ by adding a new vertex $s$ and connecting $s$ to all the four vertices. Let $\mathcal{P}$ be the bipartion of $C_{4}$. Then $G$ admits no complete 3 -admissible $\mathcal{P}$-allowed splitting off. The essential properties of this example are kept in a more general structure called $C_{4}$-obstacle, defined as follows.

A partition $\left\{A_{1}, A_{2}, A_{3}, A_{4}\right\}$ of $V$ is called a $\boldsymbol{C}_{4}$-obstacle of $G$ if $k$ is odd and

$$
\begin{aligned}
d\left(A_{i}\right) & =k & & \forall 1 \leq i \leq 4, \\
d\left(A_{i}, A_{i+2}\right) & =0 & & \forall 1 \leq i \leq 2, \\
\left|P_{l}\right| & =\frac{d(s)}{2} & & \exists 1 \leq l \leq r, \\
\delta\left(A_{j} \cup A_{j+2}\right) \cap \delta(s) & =P_{l} & & \exists 1 \leq j \leq 2 .
\end{aligned}
$$

Another difficulty may turn up if the partition contains more than two members. Suppose we wish to make 3 -edge-connected a 6 -cycle $C_{6}$. Clearly, this can be done by adding three edges. Note that, though the optimal solution is not unique, we have to add at least one diagonal edge. Now suppose that we have the additional condition that we must maintain the non-adjacency of the opposite vertices of $C_{6}$. Then this solution is not feasible any more. In this case we have to add 4 edges. Let us reformulate this difficulty in terms of splitting off. Let $G$ be the graph obtained from $C_{6}$ by adding a new vertex $s$ and connecting $s$ to all the six vertices. Let $\mathcal{P}$ the 3-partion of $C_{6}$ where the sets consist of the opposite vertices of $C_{6}$. Then $G$ admits no complete 3 -admissible $\mathcal{P}$-allowed splitting off. As before, the essential properties of this example are kept in a more general structure called $C_{6}$-obstacle, defined as follows.

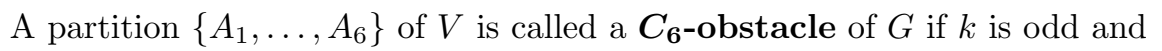

$$
\begin{aligned}
d\left(A_{i}\right) & =k & & \forall 1 \leq i \leq 6, \\
d\left(A_{i}, A_{i+1}\right) & =\frac{k-1}{2} & & \forall 1 \leq i \leq 6,\left(A_{7}=A_{1}\right) \\
d\left(s, A_{i}\right) & =1 & & \forall 1 \leq i \leq 6,
\end{aligned}
$$




$$
\delta\left(A_{j} \cup A_{j+3}\right) \cap \delta(s)=P_{l_{j}} \quad \forall 1 \leq j \leq 3, \exists 1 \leq l_{j} \leq r .
$$

We must emphasize that these difficulties may exist only if the target edge-connectivity is odd, and if one of them exists, then no complete allowed splitting off may exist. Now we are in a position to provide a characterization of the existence of a complete allowed splitting off.

Theorem 14 (Bang-Jensen, Gabow, Jordán, Szigeti [2]). Let $G=(V+$ $s, E)$ be a graph with $d(s)$ even, $2 \leq k \in \mathbb{Z}$, and $\mathcal{P}=\left\{P_{1}, \ldots, P_{r}\right\}$ a partition of $V$. Then there exists a complete $k$-admissible $\mathcal{P}$-allowed splitting off at $s$ if and only if

$$
\begin{array}{r}
G \text { is } k \text {-edge-connected in } V, \\
d\left(s, P_{i}\right) \leq \frac{d(s)}{2} \quad \forall 1 \leq i \leq r, \\
G \text { contains no } C_{4} \text { - or } C_{6} \text {-obstacle. }
\end{array}
$$

In the special case, when each element of $\mathcal{P}$ is a singleton, no $C_{4}$ or $C_{6^{-}}$ obstacle can exist, thus Theorem 14 implies Theorem 2(a), while when $|\mathcal{P}|=$ 2 , it provides (with Theorem 1) a solution for the problem of global edgeconnectivity augmentation in bipartite graphs. We may observe that in this case no $C_{6}$-obstacle can exist.

The following result from [35] is a slight generalization of Theorem 14. The motivation of this form is that it allows us to contract tight sets and hence it enables us to simplify the proof that will be presented in Section 1.8.2.

Theorem 15 (Szigeti [35]). Let $G=(V+s, E)$ be a graph with $d(s)$ even, $2 \leq k \in \mathbb{Z}$, and $\mathcal{P}=\left\{P_{1}, \ldots, P_{r}\right\}$ a partition of $\delta(s)$. Then there exists a complete $k$-admissible $\mathcal{P}$-allowed splitting off at $s$ if and only if (1.34), (1.36) and the following condition are satisfied.

$$
\left|P_{i}\right| \leq \frac{d(s)}{2} \quad \forall 1 \leq i \leq r
$$

\section{Augmentation of global edge-connectivity with partition constraints}

We present a more precise reformulation of the problem of this section: Given a graph $G=(V, E)$, an integer $k$ and a partition $\mathcal{P}=\left\{P_{1}, \ldots, P_{r}\right\}$ of $V$, what is the minimum number $\boldsymbol{O P} \boldsymbol{T}_{\mathcal{P}}^{\boldsymbol{k}}$ of $\mathcal{P}$-allowed edges whose addition results in a $k$-edge-connected graph?

Note that, by taking the partition of all singletons in this problem, we get as a special case the global edge-connectivity augmentation problem in graphs. where

The following theorem answers this problem. Let $\boldsymbol{\Phi}:=\max \left\{\alpha, \beta_{1}, \ldots, \beta_{r}\right\}$ 


$$
\begin{aligned}
\alpha & :=\max \left\{\left\lceil\sum_{X \in \mathcal{X}} \frac{k-d(X)}{2}\right\rceil: \mathcal{X} \in \mathcal{S}(V)\right\}, \\
\beta_{j} & :=\max \left\{\sum_{Y \in \mathcal{Y}}(k-d(Y)): \mathcal{Y} \in \mathcal{S}\left(P_{j}\right)\right\} \quad \forall 1 \leq j \leq r .
\end{aligned}
$$

It is crucial to point out that $\Phi$ is a lower bound for the optimal value, that is,

$$
O P T_{\mathcal{P}}^{k} \geq \Phi
$$

Indeed, by adding an edge to $G$ we may decrease the deficiency $k-d(Z)$ of at most two sets in $\mathcal{X}$ and of at most one set in each $\mathcal{Y}$, and hence we can decrease $\Phi$ by at most one.

We will have equality in (1.38), unless $G$ contains one of the following two configurations. These are the structures that force us to have a $C_{4^{-}}$or $C_{6}$-obstacle in any optimal extension.

A partition $\left\{A_{1}, A_{2}, A_{3}, A_{4}\right\}$ of $V$ is called a $C_{4}$-configuration of $G$ if $k$ is odd and

$$
\begin{aligned}
d\left(A_{i}\right) & <k & & \forall 1 \leq i \leq 4, \\
d\left(A_{i}, A_{i+2}\right) & =0 & & \forall 1 \leq i \leq 2, \\
\sum_{X \in \mathcal{X}_{i}}(k-d(X)) & =k-d\left(A_{i}\right) & & \exists \mathcal{X}_{i} \in \mathcal{S}\left(A_{i}\right) \forall 1 \leq i \leq 4, \\
\mathcal{X}_{j} \cup \mathcal{X}_{j+2} & \in \mathcal{S}\left(P_{l}\right) & & \exists 1 \leq l \leq r \exists 1 \leq j \leq 2, \\
k-d\left(A_{i}\right)+k-d\left(A_{i+2}\right) & =\Phi & & \forall 1 \leq i \leq 2 .
\end{aligned}
$$

A partition $\left\{A_{1}, A_{2}, \ldots, A_{6}\right\}$ of $V$ is called a $\boldsymbol{C}_{\mathbf{6}}$-configuration of $G$ if $k$ is odd and

$$
\begin{array}{rlrl}
d\left(A_{i}\right) & =k-1 & & \forall 1 \leq i \leq 6, \\
d\left(A_{i}, A_{i+1}\right) & =\frac{k-1}{2} & & \forall 1 \leq i \leq 6,\left(A_{7}=A_{1}\right) \\
\Phi & =3 & & \forall 1 \leq i \leq 6, \\
d\left(A_{i}^{\prime}\right) & =k-1 & & \exists 1 \leq j_{1}, j_{2}, j_{3} \leq r \forall 1 \leq i \leq 6 \\
& & \exists A_{i}^{\prime} \subseteq A_{i} \cap P_{j_{i-3\left\lfloor\frac{(i-1)}{3}\right\rfloor},}
\end{array}
$$

where the $j_{i}$ 's must be different in (1.47).

Since these configurations force us to have an obstacle in any optimal extension, in which case there exists no complete allowed splitting off in the extended graph, the existence of a configuration implies that the optimal solution must contain at least $\Phi+1$ edges. We show that this can be achived. Using Theorems 1 and 14 we can prove with some effort the following result. 
Theorem 16 (Bang-Jensen, Gabow, Jordán, Szigeti [2]). Let $G=$ $(V, E)$ be a connected graph, $\mathcal{P}=\left\{P_{1}, \ldots, P_{r}\right\}$ a partition of $V$ and $k \geq 2$. Then $G$ can be made $k$-edge-connected by adding $\Phi \mathcal{P}$-allowed edges (that is $\left.O P T_{\mathcal{P}}^{k}=\Phi\right)$ unless $G$ contains a $C_{4}$ - or $C_{6}$-configuration when we need one more edge (that is $O P T_{\mathcal{P}}^{k}=\Phi+1$ ).

As the global edge-connectivity augmentation problem without partition constraints can be considered as one with partition constraints where each element of $\mathcal{P}$ is a singleton, in which case no $C_{4^{-}}$or $C_{6}$-configuration can exist and $\Phi=\alpha$, Theorem 16 implies Theorem 3. We note that if we want to augment the global edge-connectivity of a bipartite graph then $|\mathcal{P}|=2$ and hence a $C_{6}$-configuration can not exist.

\subsection{Hypergraphs}

In this section we wish to present problems on edge-connectivity augmentation in hypergraphs and their solutions. We continue with the same structure as before: each subsection is devoted to one problem, and it is divided in two parts; results on splitting off and then the minimax result on augmentation. We mention that some of the problems that were easy in graphs turn out to be already too difficult in hypergraphs.

\subsubsection{Augmentation of global edge-connectivity in a hypergraph by adding graph edges}

In this section we consider the problem of global edge-connectivity augmentation in hypergraphs by adding graph edges, that can be formulated as follows: Given a hypergraph $\mathcal{G}$ and $k \in \mathbb{Z}^{+}$, what is the minimum number of graph edges (hyperedges of size two) whose addition results in a $k$-edge-connected hypergraph?

Of course, if the hypergraph is in fact a graph, then the problem reduces to the global edge-connectivity augmentation problem in graphs.

One of the main difficulties with hypergraphs that we have to handle the case when $k=1$. Why is it so? Becasue by deleting a hyperedge the number of connected components may increase by a large value. This discussion shows the necessity of condition (1.49).

The corresponding splitting off result is the following.

Theorem 17 (Bang-Jensen, Jackson [3]). Let $\mathcal{G}=(V+s, \mathcal{E})$ be a hypergraph and $\gamma, k \in \mathbb{Z}^{+}$so that $d(s)=2 \gamma$ and each edge incident to $s$ is of size two. Then there is a complete $k$-admissible splitting off at $s$ if and only if

$$
\begin{aligned}
& \mathcal{G} \text { is k-edge-connected in } V, \\
& c(\mathcal{G}-s-\mathcal{H})-1 \leq \gamma \forall \mathcal{H} \subseteq \mathcal{E},|\mathcal{H}| \leq k-1 .
\end{aligned}
$$


If $\mathcal{G}$ is a graph, then the second condition of the above theorem is satisfied (after deleting $k-1$ edges in a $k$-edge-connected graph, the graph remains connected) and hence Theorem 17 implies Theorem 2(a).

Theorems 1 and 17 imply the following theorem that solves the problem of this section. We notice that (1.50) and the fact that the function $k-d_{\mathcal{H}}(X)$ is symmetric skew-supermodular guarantees that the optimal extension (with respect to $k$ ) can be made by $2 \gamma$ edges and (1.51) allows us to find the complete $k$-admissible splitting off.

Theorem 18 (Bang-Jensen, Jackson [3]). Let $\mathcal{G}$ be a hypergraph and $k \in$ $\mathbb{Z}^{+}$. Then $\mathcal{G}$ can be made $k$-edge-connected by adding at most $\gamma$ new edges (hyperedges of size two) if and only if

$$
\begin{aligned}
\sum_{X \in \mathcal{X}}\left(k-d_{\mathcal{G}}(X)\right) \leq 2 \gamma \quad \forall \mathcal{X} \in \mathcal{S}(V), \\
c(\mathcal{G}-\mathcal{H})-1 \leq \gamma \quad \forall \mathcal{H} \subseteq E(\mathcal{G}),|\mathcal{H}| \leq k-1 .
\end{aligned}
$$

As above, if $\mathcal{G}$ is a graph, then (1.51) is implied by (1.50) and hence Theorem 18 implies Theorem 3.

\subsubsection{Augmentation of global edge-connectivity of a hypergraph by adding a uniform hypergraph}

As a natural generalization of the problem of the previous section, one can consider the global edge-connectivity augmentation problem of a hypergraph by adding hyperedges of the same size, namely: Given a hypergraph $\mathcal{G}$ and $k, r \in \mathbb{Z}^{+}$, what is the minimum number of hyperedges of size $r$ whose addition results in a $k$-edge-connected hypergraph?

Since the function $k-d_{\mathcal{H}}(X)$ is symmetric crossing supermodular, the problem of covering a symmetric crossing supermodular function by a uniform hypergraph contains this problem as a special case, thus Theorem 26 implies the following theorem. We mention that it is not easy to see that (1.54) implies (1.65) when $p(X)=k-d_{\mathcal{G}}(X)$.

Theorem 19 (T. Király [26]). Let $\mathcal{G}=(V, \mathcal{E})$ be a hypergraph and $k, r \in$ $\mathbb{Z}_{+}, r \leq|V|$. Then $\mathcal{G}$ can be made $k$-edge-connected by adding at most $\gamma$ new hyperedges of size $r$ if and only if

$$
\begin{array}{rlrl}
\sum_{X \in \mathcal{X}}\left(k-d_{\mathcal{G}}(X)\right) & \leq r \gamma & & \forall \mathcal{X} \in \mathcal{S}(V), \\
k-d_{\mathcal{G}}(X) & \leq \gamma & & \forall X \subseteq V, \\
c(\mathcal{G}-\mathcal{H})-1 & \leq(r-1) \gamma & \forall \mathcal{H} \subseteq \mathcal{E},|\mathcal{H}|=k-1 .
\end{array}
$$

The special case when we want to augment the edge-connectivity from $k$ to $k+1$ was solved earlier in Fleiner, Jordán [13]. Note that, when $r=2$, then (1.52) and (1.54) reduce to (1.50) and (1.51), and (1.53) is implied by (1.52), so Theorem 19 implies Theorem 18. 


\subsubsection{Augmentation of local edge-connectivity of a hypergraph by} adding graph edges

This section is the devoted to the following problem.

Hypergraph Local Edge-Connectivity Augmentation by a Graph Instance: A hypergraph $\mathcal{H}$ on $V$, a symmetric requirement function $r(u, v) \in$ $\mathbb{Z} \forall u, v \in V$, and $\gamma \in \mathbb{Z}_{+}$.

Question: Does there exist a graph $G=(V, E)$ with at most $\gamma$ edges so that $\lambda_{\mathcal{H}+G}(u, v) \geq r(u, v) \forall u, v \in V ?$

The following theorem shows that this problem is already too complicated.

Theorem 20 (Cosh, Jackson, Z. Király [11]). The problem HYPERgraph Local Edge-Connectivity Augmentation by a Graph is NPcomplete.

We mention that the above problem remains NP-complete if the hypergraph contains only just one hyperedge of size greater than 2 .

We remark that the special case of the problem HYPERGRAPH LOCAL Edge-Connectivity Augmentation By a Graph for 2-3 hypergraphs is tractable. This is because of the fact that a 2-3 hypergraph can be transformed into a graph with the same local edge-connectivities and vice versa. Thus Theorem 12 implies the following.

Theorem 21 (Jordán, Szigeti [24]). Let $r$ be a requirement function with $2 \leq r(u, v) \in \mathbb{Z} \quad \forall u, v \in V$. A 2-3 hypergraph $G=(V, E)$ can be made $\boldsymbol{r}$-edgeconnected by adding $\gamma$ edges and $\gamma^{\prime} 3$-hyperedges if and only if

$$
\begin{gathered}
\sum_{X \in \mathcal{X}}(R(X)-d(X)) \leq 2 \gamma+3 \gamma^{\prime} \quad \forall \mathcal{X} \in \mathcal{S}(V), \\
G \text { is } \mathbf{r}-\left(\gamma+\gamma^{\prime}\right) \text {-edge-connected. }
\end{gathered}
$$

\subsubsection{Bipartite constrained augmentation of local edge-connectivity of a hypergraph by adding graph edges}

In this section we consider a restricted version of the main problem of the preceding section.

Bipartition Constrained Hypergraph Local Edge-Connectivity Augmentation By a Graph

Instance: A hypergraph $\mathcal{H}$ on $V$, a bipartition $\{A, B\}$ of $V$, a symmetric requirement function $r(u, v) \in \mathbb{Z} \forall u, v \in V$, and $\gamma \in \mathbb{Z}_{+}$.

Question: Does there exist a bipartite graph $G=(A, B ; E)$ with colour classes $A$ and $B$ with at most $\gamma$ edges so that $\lambda_{\mathcal{H}+G}(u, v) \geq r(u, v) \forall u, v \in V$ ?

By checking the proof of Theorem 20 given in [11] we may observe that it also provides the NP-completeness of the above problem.

Theorem 22. The problem Bipartition Constrained Hypergraph LoCal Edge-Connectivity Augmentation by a Graph is NP-complete. 


\subsubsection{Augmentation of local edge-connectivity of a hypergraph by adding hyperedges}

In this section we want to augment a hypergraph by adding hyperedges to satisfy local edge-connectivity requirements. The problem of minimizing the number of hyperedges is trivial since we can add the whole vertex set as many times as needed. What we want to minimize is the total size of the hypergraph to be added, that is, the sum of the sizes of the hyperedges. More precisely, the local edge connectivy augmentation problem of a hypergraph by adding hyperedges is the following: Given a hypergraph $\mathcal{G}$ and a symmetric requirement function $r: V \times V \rightarrow \mathbb{Z}_{+}$, what is the minimum total size $\sum_{H \in \mathcal{H}}|H|$ of new hyperedges $H \in \mathcal{H}$ whose addition results in an $\mathbf{r}$-edge-connected hypergraph?

Recall that $R(X)=\max \{r(x, y): x \in X, y \in V-X\}$ and that the function $R(X)-d_{\mathcal{G}}(X)$ is symmetric skew-supermodular. Then the problem of covering a symmetric skew-supermodular function by a hypergraph contains this problem as a special case thus Theorem 31 implies the following theorem.

Theorem 23 (Szigeti [31]). Let $\mathcal{G}$ be a hypergraph on $V$ and $r(u, v) \in$ $\mathbb{Z}_{+} \forall u, v \in V$ a symmetric requirement function. Then there exists a hypergraph $\mathcal{H}$ on $V$ with $\sum_{H \in \mathcal{H}}|H| \leq \gamma$ so that $\lambda_{\mathcal{G}+\mathcal{H}}(u, v) \geq r(u, v) \forall u, v \in V$ if and only if

$$
\sum_{X \in \mathcal{X}}\left(R(X)-d_{\mathcal{G}}(X)\right) \leq \gamma \quad \forall \mathcal{X} \in \mathcal{S}(V)
$$

\subsection{Abstract forms}

In this section we present results on "connectivity" set functions, that generalize the results presented in the previous sections. We start with a generalization of splitting off. Let $p: 2^{V} \rightarrow \mathbb{Z}$ be a function and $H=(V+s, E)$ a graph so that each edge is incident to $s$. By a complete hypergraph splitting off we mean a hypergraph $\mathcal{H}$ on $V$ such that $\mathcal{H}$ covers $p$ and the following is satisfied:

$$
d_{\mathcal{H}}(v)=d_{H}(v) \quad \forall v \in V .
$$

In other words, it is a degree constrained hypergraph that covers $p$. Note that there is no restriction on the size of the hyperedges. If we pose the restriction that each hyperedge must be of size at most two, then we are back to the definition of the usual complete splitting off.

\subsubsection{Symmetric crossing supermodular functions}

The problem of covering a symmetric crossing supermodular function can be considered as a generalization of the global edge-connectivity augmentation 
problem. In this section we present the result of Benczúr and Frank on covering a symmetric crossing supermodular function by a graph (that generalizes Theorem 17) and then its generalization due to T. Király on covering such a function by an $r$-uniform hypergraph (that also generalizes Theorem 19).

\section{Complete uniform hypergraph splitting off}

The splitting off results that will solve (together with Theorem 1) the above mentioned problems are the following. We call the attention to the fact that in Theorem 24 a hyperedge may contain the same vertex many times and the size of the hyperedge is meant by multiplicities.

A partition $\left\{V_{1}, \ldots, V_{l}\right\}$ of $V$ is called an $\boldsymbol{l}$-partition. Let $r \in \mathbb{Z}_{+}$, $H=(V+s, E)$ a graph so that each edge is incident to $s$ and $r$ divides $d_{H}(s)$ and $p: 2^{V} \rightarrow \mathbb{Z}_{+}$a set function. An $l$-partition is called p-full if $l>r$ and $p\left(\bigcup_{i \in I} V_{i}\right)>0 \forall \emptyset \neq I \subset\{1, \ldots, l\}$. A $p$-full partition is called deficient if $\frac{l-1}{r-1}>\frac{d_{H}(s)}{r}$. We say that the set function $p$ is positively crossing supermodular if (1.10) is satisfied if $X, Y \subseteq V$ are crossing with $p(X), p(Y)>0$.

Theorem 24 (T. Király [26]). Let $p: 2^{V} \rightarrow \mathbb{Z}_{+}$be a symmetric, positively crossing supermodular set function, $r \geq 2$ an integer, and $H=(V+s, E)$ a graph so that each edge is incident to $s$ with $r$ divides $d_{H}(s)$. Then there exists a complete r-uniform hypergraph splitting off if and only if

$$
\min \left\{d_{H}(X), d_{H}(s) / r\right\} \geq p(X) \forall X \subseteq V,
$$

There are no deficient partitions.

The special case when $r=2$ was proved earlier by Benczúr and Frank [6].

\section{Covering a symmetric crossing supermodular function by a graph}

As a generalization of the global edge-connectivity augmentation in hypergraphs by adding graph edges, Benczúr and Frank considered the problem of covering a symmetric crossing supermodular function by a graph, namely: Given a symmetric, positively crossing supermodular set function $p$, what is the minimum number of edges that cover $p$ ?

As a generalization of Theorem 18 they proved in [6] the following result by applying Theorem 1 and their splitting off result which is the $r=2$ special case of Theorem 24 .

Theorem 25 (Benczúr, Frank [6]). Let $p: 2^{V} \rightarrow \mathbb{Z}_{+}$be a symmetric, positively crossing supermodular set function. Then there exists a graph on $V$ with $\gamma$ edges that covers $p$ if and only if

$$
\begin{aligned}
\sum_{X \in \mathcal{X}} p(X) & \leq 2 \gamma \forall \mathcal{X} \in \mathcal{S}(V), \\
l-1 & \leq \gamma \quad \text { if a } p \text {-full l-partition exists. }
\end{aligned}
$$


For a hypergraph $\mathcal{G}$, the function $k-d_{\mathcal{G}}(X)$ is symmetric, positively crossing supermodular, thus Theorem 25 implies Theorem 18.

\section{Covering a symmetric crossing supermodular function by a uniform hypergraph}

As a generalization of the problems of covering a symmetric crossing supermodular function by graph edges and of the global edge-connectivity augmentation in hypergraphs by $r$-uniform hyperedges, T. Király considered the problem of covering a symmetric crossing supermodular function by an $r$-uniform hypergraph, namely: Given a symmetric, positively crossing supermodular set function $p$ and $r \in \mathbb{Z}^{+}$, what is the minimum number of hyperedges of size $r$ that cover $p$ ?

As a generalization of Theorems 25 and 19 he obtained in [26] the following result by applying Theorems 1 and 24 .

Theorem 26 (T. Király [26]). Let $p: 2^{V} \rightarrow \mathbb{Z}_{+}$be a symmetric, positively crossing supermodular set function, $2 \leq r \leq|V|$ an integer. Then there exists an $r$-uniform hypergraph on $V$ with $\gamma$ hyperedges that covers $p$ if and only if

$$
\begin{aligned}
\sum_{X \in \mathcal{X}} p(X) & \leq r \gamma & & \forall \mathcal{X} \in \mathcal{S}(V), \\
p(X) & \leq \gamma & & \forall X \subseteq V, \\
l-1 & \leq(r-1) \gamma & & \text { if a } p \text {-full l-partition exists. }
\end{aligned}
$$

Note that Theorem 26 implies Theorem 25 (when $r=2$ ) and Theorem 19 (when the function is the deficiency function of a hypergraph).

\subsubsection{Symmetric skew-supermodular functions}

The problem of covering a symmetric skew-supermodular function can be considered as a common generalization of many of the preceding problems. In this section we provide a nice proof of the NP-completeness of the problem of covering a symmetric skew-supermodular function by a graph (which we know already since it generalizes the problem of local edge-connectivity augmentation of a hypergraph by graph edges) while the problem of covering such a function by a hypergraph is solvable in a certain sense.

\section{Covering a symmetric skew-supermodular function by a graph}

The problem of this section can be formulated as follows.

Minimum Cover of a Symmetric, Skew-Supermodular Function by A GRAPH

Instance: A symmetric skew-supermodular function $p$ on $V$ and $\gamma \in \mathbb{Z}^{+}$. 
Question: Does there exist a graph on $V$ with at most $\gamma$ edges that covers $p$ ?

Note that, as we already mentioned, the NP-complete problem HYPERgraph Local Edge-Connectivity Augmentation by a Graph is a special case of the problem Minimum Cover of a Symmetric, SkewSupermodular Function BY a Graph and hence this last one is also NP-complete, which we prove here in an elegant way. The proof is due to Z. Király [27] and independently to Nutov [30].

Theorem 27. [27] The problem Minimum Cover of a Symmetric, SkewSupermodular Function By a Graph is NP-complete.

Proof. [Z. Király] We reduce 3Dm to Minimum Cover of a Symmetric, Skew-Supermodular Function by a Graph. Let $\mathcal{H}$ be a 3 -uniform hypergraph on $V$. Let $n:=|V|$. Let $p(X):=1$ if $|X| \in\{1,2, n-1, n-2\}$, or $X \in \overline{\mathcal{H}}$ or $V-X \in \overline{\mathcal{H}}$ and 0 otherwise. It is easy to verify that $p(X)$ is a symmetric skew-supermodular set function. The following completes the proof. There exists a graph on $V$ with at most $2 n / 3$ edges that covers $p$ if and only if $\mathcal{H}$ contans a 3 -dimensional matching. Indeed, first suppose that $H_{1}, \ldots, H_{n / 3}$ is a 3-dimensional matching. For each $H_{i}$, let us choose two edges on $V\left(H_{i}\right)$, and let $F$ be the union of these edges. Then $|F|=2 n / 3$ and, clearly, $F$ covers $p$. Now suppose that the graph $F$ covers $p$ and $|E(F)| \leq 2 n / 3$. Let $F_{1}, \ldots, F_{l}$ be the connected components of $F$. Since $F$ covers $p,\left|V\left(F_{i}\right)\right| \geq 3$ for $1 \leq i \leq l$ thus $l \leq n / 3$. Then $2 n / 3 \geq|E(F)| \geq n-l \geq 2 n / 3$ so $l=n / 3$ and $\left|V\left(F_{i}\right)\right|=3$ for $1 \leq i \leq l$. Since $F$ covers $p$, each $F_{i}$ belongs to $\mathcal{H}$, that is, $F_{1}, \ldots, F_{l}$ is a 3-dimensional matching.

By the following theorem, which is an easy corollary of Theorem 31, Minimum Cover of a Symmetric, Skew-Supermodular Function by a GRAPH can be solved if $p(X)$ is even for every $X \subseteq V$.

Theorem 28 (Szigeti [31]). Let $p$ be a symmetric, skew-supermodular, "even integer" valued function on $V$. Then there exists a graph on $V$ with at most $\gamma$ edges that covers $p$ if and only if (1.61) is satisfied.

Proof. To prove the difficult part of Theorem 28, suppose that (1.61) is satisfied and let $p^{\prime}(X)=p(X) / 2$. Then, by the assumptions on $p, p^{\prime}$ is a symmetric, skew-supermodular, integer valued function on $V$. Note that, since $p$ satisfies (1.61), $p^{\prime}$ satisfies (1.13). Then, by Theorem 31, there exists a hypergraph $\mathcal{H}$ on $V$ so that $\sum_{H \in \mathcal{H}}|H| \leq \gamma$ and $\mathcal{H}$ covers $p^{\prime}$, that is, $d_{\mathcal{H}}(X) \geq p^{\prime}(X)$. Let $G:=\left(V, \cup_{H \in \mathcal{H}} E_{H}\right)$ where $E_{H}$ is an arbitrary cycle on $V(H)$ for each $H \in \mathcal{H}$. Then $|E(G)|=\sum_{H \in \mathcal{H}}\left|E_{H}\right|=\sum_{H \in \mathcal{H}}|H| \leq \gamma$ and $d_{G}(X)=2 d_{\mathcal{H}}(X) \geq 2 p^{\prime}(X)=p(X)$, that completes the proof.

Note that the problem Minimum Cover of a Symmetric, SkewSuPERMOdUlar Function BY a GRAPH contains as a special case : the 
global and local edge-connectivity augmentation in graphs and in hypergraphs, the global edge-connectivity augmentation over symmetric parity families, the node to area edge-connectivity augmentation, and the problem of covering a symmetric crossing supermodular function by a graph.

\section{Covering a symmetric semi-monotone function by a graph}

In this section, we mention another special case of the NP-complete problem Minimum Cover of a Symmetric, Skew-Supermodular Function by A GRAPH, that can be solved in polynomial time.

We call a function $P: 2^{V} \rightarrow \mathbb{Z}$ semi-monotone if $P(\emptyset)=P(V)=0$ and for each set $\emptyset \neq X \neq V, 0 \leq P(X) \leq P\left(X^{\prime}\right)$ either for all $\emptyset \neq X^{\prime} \subseteq X$ or for all $\emptyset \neq X^{\prime} \subseteq V-X$. We note that a symmetric semi-monotone function is skew-supermodular.

We consider the problem of Covering a symmetric semi-monotone function by a graph: Given a graph $G=(V, E)$ and a symmetric semimonotone function $P$ on $V$, add a minimum number $\boldsymbol{O p t}(\boldsymbol{P}, \boldsymbol{G})$ of new edges to $G$ to get a covering of $P$.

We have already seen in Section 1.4.4 that the node to area global edgeconnectivity augmentation problem is a special case of this problem. On the other hand, any instance of the symmetric semi-monotone function covering problem can easily be formulated as a node to area global edge-connectivity augmentation problem. This discussion establishes the surprising equivalence between two seemingly not closely related problems, showing that these are two alternative models of the same problem. (see [20], [18])

We have to emphasize that this problem in general is NP-complete because the function defined in the proof of Theorem 27 is semi-monotone. However, if the function does not take the value 1 , then it can be solved.

Let us define $Q(G):=\max \left\{\sum_{X \in \mathcal{X}} q(X): \mathcal{X} \in \mathcal{S}(V)\right\}$, where $q(X)=$ $P(X)-d_{G}(X)$. Now we have a lower bound for the optimal value, namely $\operatorname{Opt}(P, G) \geq\left\lceil\frac{Q(G)}{2}\right\rceil$. Usually equality will hold, unless the graph contains a special configuration.

Since the above defined function $q(X)$ is symmetric skew-supermodular, Theorem 1 and a suitable splitting result [18] provide the solution of the covering problem.

Theorem 29 (Ishii [20], Grappe and Szigeti [18]). Let $G=(V, E)$ be a graph and $P$ a symmetric semi-monotone function on $V$ so that $P(X) \neq$ $1 \forall X \subseteq V$. If $G$ contains no configuration, then $O p t(P, G)=\left\lceil\frac{Q(G)}{2}\right\rceil$, otherwise $\operatorname{Opt}(P, G)=\left\lceil\frac{Q(G)}{2}\right\rceil+1$.

The definition of the configuration (which is fairly complicated) and a short proof of Theorem 29 can be found in [18]. Notice that Theorem 29 implies Theorem 9. 


\section{Complete hypergraph splitting off}

For symmetric skew-supermodular functions we have the following splitting off result.

Theorem 30 (Szigeti [31]). Let $p: 2^{V} \rightarrow \mathbb{Z}$ be a symmetric skewsupermodular function. Let $H=(V+s, E)$ be a graph so that each edge is incident to $s$. Then there exists a complete hypergraph splitting off if and only if $H$ covers $p$.

T. Király [25] has recently found a very short proof for a slight extension of Theorem 30.

\section{Covering a symmetric skew-supermodular function by a hypergraph}

In this section we provide the solution for the problem of covering a symmetric skew-supermodular function by a hypergraph, that is, a generalization of the problem of hypergraph local edge-connectivity augmentation by hyperedges, and that can be formulated as follows: Given a symmetric skew-supermodular function $p$, what is the minimum total size $\sum_{H \in \mathcal{H}}|H|$ of a hypergraph $\mathcal{H}$ that covers $p$ ?

Theorems 1 and 30 provide the following generalization of Theorem 23.

Theorem 31 (Szigeti [31]). Let $p: 2^{V} \rightarrow \mathbb{Z}$ be a symmetric skewsupermodular function. Then there exists a hypergraph $\mathcal{H}$ on $V$ with $\sum_{H \in \mathcal{H}}|H|$ $\leq \gamma$ so that $\mathcal{H}$ covers $p$ if and only if (1.13) is satisfied.

Note that only the total size of the hypergraph is guaranteed and no information is available on the size of the hyperedges. This is not a surprise in the light of the NP-completeness of the problem Minimum Cover of a Symmetric, Skew-Supermodular Function by a Graph. However, Bernáth and T. Király [7] have recently observed that the hypergraph $\mathcal{H}$ in Theorem 31 can be chosen so that each edge except one is of size two. They also proved that the hypergraph can be chosen so that each edge is of size $k$ or $k+1$ for some $2 \leq k \in \mathbb{Z}$.

\subsection{Open problems}

In this section we pose some open problems related to (more precisely: generalizing of) the problems of this paper. 


\subsubsection{Graphs}

The following open problem, called node to area local edge-connectivity augmentation and mentioned in [19], is a natural generalization of the node to area global edge-connectivity augmentation problem: Given a graph $G=$ $(V, E)$, a family $\mathcal{W}$ of sets $W \subseteq V$, and a requirement function $r: \mathcal{W} \times V \rightarrow$ $\mathbb{Z}_{+}$, add a minimum number of new edges to $G$ so that the resulting graph contains $r(W, v)$ edge-disjoint paths from any area $W \in \mathcal{W}$ to any vertex $v \notin W$.

A result on partition constrained detachment preserving global edge-connectivity would imply Theorems 15 and 10 . To be more precise we can consider the following problem: Given a graph $G=(V+s, E)$, a partition $\mathcal{P}$ of $\delta(s)$, a degree specification $f(s)$ and a positive integer $k$, decide whether $G$ has an $f(s)$-detachment that is $k$-edge-connected in $V$ and $\mathcal{P}$-allowed (meaning that for each new vertex $s_{i}$, the edges incident to $s_{i}$ belong to different members of $\mathcal{P}$ ).

A characterization of the existence of a partition constrained complete splitting off satisfiying a requirement function would imply Theorem 14 and also Theorem 12 as it was observed by Frank [17]. Indeed, for a graph $G=$ $(V+s, E)$, a requirement function $r: V \times V \rightarrow \mathbb{Z}_{+}(r(u, v) \geq 2 \forall u, v \in V)$ and a degree specification $f(s)=\left(d_{1}, \ldots, d_{p}\right)\left(d_{i} \geq 2 \forall i\right)$, let $G^{\prime}$ be obtained from $G$ by adding $p$ new vertices $s_{1}, \ldots, s_{p}$ and connecting each vertex $s_{i}$ to $s$ by $d_{i}$ new edges, let $r^{\prime}(u, v):=r(u, v)$ if $u, v \in V$ and 2 if $\{u, v\} \cap\left\{s_{1}, \ldots s_{p}\right\} \neq \emptyset$ and let $\mathcal{P}:=\left\{V,\left\{s_{1}, \ldots, s_{p}\right\}\right\}$. Then $G^{\prime \prime}$ is a complete $\boldsymbol{r}^{\prime}$-edge-connected $\mathcal{P}$-allowed splitting off of $G^{\prime}$ if and only if $G^{\prime \prime}$ is a $\boldsymbol{r}$-edge-connected $f(s)$-detachment of G.

Note that the complexity of the local edge-connectivity augmentation problem in a graph with bipartition constraint is not known, however, as we have seen, the problem BIPARTITION CONSTRAINED HyPERGRAPH LOCal Edge-Connectivity Augmentation by a Graph is NP-complete.

A common generalization of the above two problems is the problem of the existence of a partition constrained detachment satisfying a requirement function.

\subsubsection{Hypergraphs}

The following problem of global edge-connectivity augmentation in hypergraphs with hyperedges of given sizes is still open: Given a hypergraph $\mathcal{G}$ and $k, d_{1}, \ldots, d_{p} \in \mathbb{Z}^{+}$decide whether it is possible to add $p$ hyperedges of sizes $d_{1}, \ldots, d_{p}$ to $\mathcal{G}$ to get a $k$-edge-connected hypergraph. More generally we can consider the problem of covering a symmetric crossing supermodular function with hyperedges of given sizes. A solution to these problems would imply Theorems 19 and 24, respectively. 
We may also consider the problem of partition constrained complete splitting off preserving global edge-connectivity in hypergraphs: Given a hypergraph $\mathcal{G}=(V+s, E)$ such that $\mathcal{G}$ is $k$ edge-connected in $V$ and no hyperedge of size at least three is incident to $s$, and a partition $\mathcal{P}$ of $\delta(s)$, decide whether there exists a $\mathcal{P}$-allowed $k$-admissible complete splitting off at $s$. A result on this problem would imply Theorem 14 . The bipartite case was done by Cosh [10].

The following problem of detachment preserving global edge-connectivity in hypergraphs is also open: Given a hypergraph $\mathcal{G}=(V+s, E)$ such that $\mathcal{G}$ is $k$-edge-connected in $V$ and no hyperedge of size at least three is incident to $s$, and a degree specification $f(s)$, decide whether there exists an $f(s)$-detachment such that the resulting hypergraph is $k$-edge-connected in $V$. A result on this problem would imply Theorems 10 and 17 . The case of local edge-connectivity (being a generalization of the problem HYPERGRAPH LOcal Edge-Connectivity Augmentation by a Graph) is NP-complete.

\subsection{Appendix}

\subsubsection{A short proof of Theorem 12}

The following short proof is from [34]. Note that, by Menger's Theorem, $G$ is $r$-edge-connected if and only if $h_{G}^{r}(X) \geq 0 \forall X \subseteq V$, where $h_{G}^{r}(X):=$ $d_{G}(X)-R(X)$. The following basic property of the function $h$ follows from the facts that $d_{G}(X)$ satisfies both (1.2) and (1.3) and that $R(X)$ is skewsupermodular, and will be used frequently in this section. For any two subsets $X, Y \subseteq V$ at least one of (1.66) and (1.67) holds. If $X \cup Y=V$ then (1.67) always holds (with equality).

$$
\begin{aligned}
& h_{G}^{r}(X)+h_{G}^{r}(Y) \geq h_{G}^{r}(X \cap Y)+h_{G}^{r}(X \cup Y)+2 d_{G}(X, Y), \\
& h_{G}^{r}(X)+h_{G}^{r}(Y) \geq h_{G}^{r}(X-Y)+h_{G}^{r}(Y-X)+2 \bar{d}_{G}(X, Y) .
\end{aligned}
$$

\section{Proof of the necessity of Theorem 12}

Let $G^{\prime}:=\left(V+\left\{s_{1}, \ldots, s_{p}\right\}, E\right)$ be an $\mathbf{r}$-edge-connected $f(s)$-detachment of $G$ at $s$. By (1.4), applied for $X=\left\{s_{1}, \ldots, s_{p}\right\},(1.22)$ is satisfied since $G^{\prime} / X=$ $G$. By (1.5), applied for every vertex $s_{i} 1 \leq i \leq p,(1.23)$ is satisfied since $G^{\prime}-X=G-s$.

\section{Proof of the sufficiency of Theorem 12}

Wlog. $p \geq 2$ and $\varphi \geq 2$. We will use induction on $z(G):=|V|+d_{G}(s)$. As we already mentioned, (1.22) and (1.23) can be reformulated as (1.68) and (1.69). Note that (1.70) holds. 


$$
\begin{aligned}
h_{G}^{r}(X) & \geq 0 \quad \forall X \subseteq V, \\
h_{G-s}^{r-\varphi}(X) & \geq 0 \quad \forall X \subseteq V . \\
h_{G-s}^{r-\varphi}(X) & =h_{G}^{r}(X)-d_{G}(s, X)+\varphi \quad \forall X \subseteq V .
\end{aligned}
$$

Lemma 2. We may assume that

$$
\text { every set } \emptyset \neq X \subset V \text { with } h_{G}^{r}(X)=0 \text { is a singleton. }
$$

Proof. Suppose there exists a set $Q$ with $h_{G}^{r}(Q)=0$ and $|Q|>1$. Then let $\hat{G}:=(\hat{V}, \hat{E})$ be obtained from $G$ by contracting $Q$ into a vertex $q$ and let $\hat{r}(u, v):=r(u, v)$ if $u, v \in \hat{V}-q$, and $\max \{r(w, x): w \in Q\}$ if $q \in\{u, v\}$ where $x=\{u, v\}-q$. It can be verified easily that $\hat{R}(\hat{X})=R(X) \forall \hat{X} \subseteq \hat{V}$, so (1.68) and (1.69) are satisfied for $\hat{G}$ and $\hat{r}$. Since $|Q|>1, z(\hat{G})<z(G)$ and hence, by induction, $\hat{G}$ has an $\hat{\boldsymbol{r}}$-edge-connected $f(s)$-detachment $\hat{G}^{\prime}$. We show that the graph $G^{\prime}$ obtained from $\hat{G}^{\prime}$ by "blowing up" $Q$ is $\boldsymbol{r}$-edge-connected and we are done. Let $X^{\prime} \subseteq V^{\prime}$. Using that $h_{G^{\prime}}^{r}(Q)=h_{G}^{r}(Q)=0$, the skew-submodularity of $h_{G^{\prime}}^{r}$ and the fact that if $X^{\prime}$ and $Q$ are not intersecting then $h_{G^{\prime}}^{r}\left(X^{\prime}\right) \geq 0$ (because if $X^{\prime} \subset Q$ then $h_{G^{\prime}}^{r}\left(X^{\prime}\right)=h_{G}^{r}(X) \geq 0$ by (1.68) and if $Q \subseteq X^{\prime}$ or $Q \cap X^{\prime}=\emptyset$ then $h_{G^{\prime}}^{r}\left(X^{\prime}\right)=h_{\hat{G}^{\prime}}^{\hat{r}^{\prime}}\left(\hat{X}^{\prime}\right) \geq 0$ since $\hat{G}^{\prime}$ is $\hat{\boldsymbol{r}}$-edge-connected) we get that $h_{G^{\prime}}^{r}\left(X^{\prime}\right) \geq 0$ as we wanted.

For $T \subset \delta_{G}(s)$, the T-split of $G$ is the $\left(|T|, d_{G}(s)-|T|\right)$-detachment $G^{\prime}$ of $G$ at $s$ where $\delta_{G^{\prime}}\left(s_{1}\right)=T$. For $X \subseteq V$, let $\boldsymbol{e}(\boldsymbol{T}, \boldsymbol{X})=\left|T \cap \delta_{G}(X)\right|$.

Lemma 3. There exists $T \subset \delta_{G}(s)$ with $|T|=3$ if $f(s)=(3,3, \ldots, 3)$ and $|T|=2$ otherwise such that the $T$-split $G^{\prime}$ of $G$ satisfies (1.72) and (1.73) where $\boldsymbol{r}^{\prime}(u, v):=r(u, v)$ if $u, v \in V$ and 2 otherwise and $V^{\prime}=V \cup s_{1}$.

$$
\begin{aligned}
G^{\prime} & \text { is } \boldsymbol{r}^{\prime} \text {-edge-connected in } V^{\prime}, \\
G^{\prime}-s & \text { is }\left(\boldsymbol{r}^{\prime}-(\boldsymbol{\varphi}-\mathbf{1})\right) \text {-edge-connected in } V^{\prime},
\end{aligned}
$$

Proof. Let $\mathcal{C}$ be defined as the minimal sets $X$ with $h_{G-s}^{r-\varphi}(X)=0$.

Claim. (1.72) and (1.73) are equivalent to

$$
\begin{array}{llrl}
h_{G}^{r}(X) & \geq 2 e(T, X)-|T| & \forall X \subset V, \\
e(T, C) \geq 1 & \forall C \in \mathcal{C} .
\end{array}
$$

Proof. (1.72) is satisfied if and only if $0 \leq h_{G^{\prime}}^{r^{\prime}}\left(X^{\prime}\right) \forall X^{\prime} \subset V^{\prime}$. Since, for $X^{\prime} \subset$ $V^{\prime}-s_{1}, h_{G^{\prime}}^{r^{\prime}}\left(X^{\prime}\right)=h_{G}^{r}(X) \geq 0,(1.72)$ is equivalent to $0 \leq h_{G^{\prime}}^{r^{\prime}}\left(X^{\prime}\right) \forall X^{\prime} \subset V^{\prime}$ containing $s_{1}$ which is, by $h_{G^{\prime}}^{r^{\prime}}\left(X^{\prime}\right)=h_{G}^{r}(X)-e(T, X)+(|T|-e(T, X))$ with $X=X^{\prime}-s_{1}$, equivalent to (1.74). (1.73) is satisfied if and only if $0 \leq h_{G^{\prime}-s}^{r^{\prime}-\varphi^{\prime}}(X)$ $\forall X \subset V^{\prime}$ not containing $s_{1}$ which is, by $h_{G^{\prime}-s}^{r^{\prime}-\varphi^{\prime}}(X)=h_{G-s}^{r-\varphi}(X)+e(T, X)-1$, equivalent to (1.75). 
Claim. The following are true for $\mathcal{C}$ and for all $C \in \mathcal{C}$ :

the sets in $\mathcal{C}$ are pairwise disjoint and $d_{G}(s, C) \geq \varphi$,

$|\mathcal{C}| \in\{0,2,3\}$, if $|\mathcal{C}|=3$ then $f(s)=(3,3, \ldots, 3)$ and $h_{G}^{r}(C)=0 .(1.77)$

Proof. By the skew-submodularity of $h_{G-s}^{r-\varphi}(X)$, the minimality of the sets in $\mathcal{C},(1.69),(1.70)$ and (1.68), (1.76) follows. If $X \in \mathcal{C}$, then $V-X$ contains a set $Y \in \mathcal{C}$ so $|\mathcal{C}| \neq 1$. By (1.76) and $d_{i} \geq 2,|\mathcal{C}| \varphi \leq \sum_{C \in \mathcal{C}} d_{G}(s, C) \leq d_{G}(s)=$ $\sum_{i=1}^{p} d_{i} \leq 3 \sum_{i=1}^{p}\left\lfloor\frac{d_{i}}{2}\right\rfloor=3 \varphi$ thus $|\mathcal{C}| \leq 3$ and if $|\mathcal{C}|=3$ then each $d_{i}=3$, that is, $f(s)=(3,3, \ldots, 3)$ and $\forall C \in \mathcal{C}, \bar{d}_{G}(s, C)=\varphi$, so by $(1.70), h_{G}^{r}(C)=0$.

By (1.77), either $|\mathcal{C}|=3$ or $|\mathcal{C}| \in\{0,2\}$. If $|\mathcal{C}|=\mathbf{3}$, then, by (1.77), $f(s)=(3,3, \ldots, 3)$. By $(1.76)$, there exists $T \subset \delta_{G}(s)$ with $|T|=3$ that satisfies (1.75). $T$ also satisfies (1.74). Indeed, by (1.77), (1.71) and (1.76), $d_{G}(s, X) \geq \varphi e(T, X)$. So, by (1.70), (1.69) and $\varphi \geq 2, h_{G}^{r}(X) \geq d_{G}(s, X)-$ $\varphi \geq \varphi(e(T, X)-1) \geq 2(e(T, X)-1) \geq 2 e(T, X)-|T|$.

From now on $|\mathcal{C}| \in\{\mathbf{0 , 2}\}$.

Claim. There exists $T=\{s u, s v\}$ that satisfies (1.74) and (1.75).

Proof. If $|\mathcal{C}|=\mathbf{0}$, then (1.75) is redundant, and, by Theorem 4(a), there is a pair $T=\{s u, s v\}$ that is $\lambda$-admissible which is equivalent to (1.74). If $\mathcal{C}=\left\{\boldsymbol{C}_{\mathbf{1}}, \boldsymbol{C}_{\mathbf{2}}\right\}$, then, by (1.76), there is a $T=\{s u, s v\}$ satisfying (1.75). $T$ also satisfies (1.74). Otherwise, there exists $u, v \in X \subset V$ with $h_{G}^{r}(X) \leq 1$. If $C_{1} \cup C_{2} \subseteq X$, then, by (1.70), (1.69), (1.76), $h_{G}^{r}(X) \geq d_{G}(s, X)-\varphi \geq$ $d_{G}\left(s, C_{1} \cup C_{2}\right)-\varphi \geq 2 \varphi-\varphi \geq 2$, contradiction, so wlog. $Y:=C_{1}-X \neq$ $\emptyset$. Since $C_{1} \in \mathcal{C}, h_{G-s}^{r-\varphi}(Y) \geq 1$. Let $d:=d_{G}\left(s, C_{1} \cap X\right)$. Then, by (1.70), $h_{G}^{r}(Y)=h_{G-s}^{r-\varphi}(Y)+d_{G}(s, Y)-\varphi \geq 1+\left(d_{G}\left(s, C_{1}\right)-d\right)-\varphi=h_{G}^{r}\left(C_{1}\right)+1-d$. If (1.67) applies for $C_{1}$ and $X$, then, by (1.68), $h_{G}^{r}(X)+h_{G}^{r}\left(C_{1}\right) \geq h_{G}^{r}(X-$ $\left.C_{1}\right)+h_{G}^{r}(Y)+2 \bar{d}_{G}\left(X, C_{1}\right) \geq h_{G}^{r}(Y)+2 d \geq h_{G}^{r}\left(C_{1}\right)+1+d \geq h_{G}^{r}\left(C_{1}\right)+2$, contradiction. So (1.66) applies for $C_{1}$ and $X$ and $Z:=C_{1} \cup X \neq V$. Since $\mathcal{C}=\left\{C_{1}, C_{2}\right\}, h_{G-s}^{r-\varphi}(Z)=h_{G-s}^{r-\varphi}(V-Z) \geq 1$. Then, by $(1.70), h_{G}^{r}(Z)=$ $h_{G-s}^{r-\varphi}(Z)+d_{G}(s, Z)-\varphi \geq 1+\left(d_{G}\left(s, C_{1}\right)+1\right)-\varphi=h_{G}^{r}\left(C_{1}\right)+2$, so, by (1.68), $h_{G}^{r}(X)+h_{G}^{r}\left(C_{1}\right) \geq h_{G}^{r}\left(X \cap C_{1}\right)+h_{G}^{r}(Z) \geq h_{G}^{r}\left(C_{1}\right)+2$, a contradiction that finishes the proof of the claim.

If $f(s) \neq(3,3, \ldots, 3)$, then, by the above claim, we are done. From now on $f(s)=(3,3, \ldots, 3)$. Then $d_{G}(s)=3 \varphi$.

Claim. $T$ can be extended to $T^{\prime} \subset \delta_{G}(s)$ with $\left|T^{\prime}\right|=3$ such that $T^{\prime}$ satisfies (1.74).

Proof. First suppose $\boldsymbol{\Gamma}(s)=\{\boldsymbol{u}, \boldsymbol{v}\}$. Since $d_{G}(s)=3 \varphi$ and $\varphi \geq 2$, wlog. $d_{G}(s, u) \geq \varphi+1$, thus there exists another copy $e^{\prime}$ of $s u$. Then, by (1.70) and (1.69), $T^{\prime}:=T \cup e^{\prime}$ satisfies (1.74). Hence $\boldsymbol{\Gamma}(\boldsymbol{s}) \neq\{\boldsymbol{u}, \boldsymbol{v}\}$. Suppose indirect that there exists a minimal set $\mathcal{M}$ of subsets of $V$ such that for 
every $z_{i} \in \Gamma(s)-\{u, v\} \neq \emptyset$ there exists a set $M_{i} \in \mathcal{M}$ violating (1.74) for $T^{\prime}:=T \cup s z_{i}$. Then, since $T$ satisfies (1.74) and by (1.71), $e\left(T^{\prime}, M_{i}\right)=3$ so $\left\{u, v, z_{i}\right\} \subseteq M_{i}$ and $h_{G}^{r}\left(M_{i}\right) \leq 2$. Clearly, $|\mathcal{M}| \geq 1$. By (1.70), (1.69), $h_{G}^{r}\left(M_{i}\right) \leq 2$ and $\varphi \geq 2$, we have $|\mathcal{M}| \geq 2$. For $M_{i}, M_{j} \in \mathcal{M}$,

$$
\begin{aligned}
h_{G}^{r}\left(M_{i}-M_{j}\right) & =0,\left(\text { so, by }(1.71), M_{i}-M_{j}=z_{i},\right) \\
\bar{d}_{G}\left(M_{i}, M_{j}\right) & =2, \\
d_{G}\left(z_{i}, M_{i}-z_{i}\right) & \geq 1 .
\end{aligned}
$$

Indeed, $2 \geq h_{G}^{r}\left(M_{i}\right), 2 \geq h_{G}^{r}\left(M_{j}\right), h_{G}^{r}\left(M_{i} \cap M_{j}\right) \geq 2 e\left(T, M_{i} \cap M_{j}\right)-|T| \geq$ $2 \times 2-2=2$ (by (1.74) and $\left.\{u, v\} \subset M_{i} \cap M_{j}\right), h_{G}^{r}\left(M_{i} \cup M_{j}\right) \geq 3$ (by the minimality of $\mathcal{M}$ ), so (1.66) cannot be satisfied for $M_{i}$ and $M_{j}$. Then $M_{i}$ and $M_{j}$ satisfy (1.67) implying (1.78) and (1.79). Moreover, since $\max \left\{R\left(z_{i}\right), R\left(M_{i}-z_{i}\right)\right\} \geq R\left(M_{i}\right)$ and $\min \left\{R\left(z_{i}\right), R\left(M_{i}-z_{i}\right)\right\} \geq 2$, we have $R\left(z_{i}\right)+R\left(M_{i}-z_{i}\right) \geq R\left(M_{i}\right)+2$, thus $2 \leq h_{G}^{r}\left(z_{i}\right)+h_{G}^{r}\left(M_{i} \cap M_{j}\right)=$ $h_{G}^{r}\left(z_{i}\right)+h_{G}^{r}\left(M_{i}-z_{i}\right) \leq h_{G}^{r}\left(M_{i}\right)-2+2 d_{G}\left(z_{i}, M_{i}-z_{i}\right) \leq 2 d_{G}\left(z_{i}, M_{i}-z_{i}\right)$ implying (1.80).

Case 1 If $\mathcal{M}=\left\{M_{1}, M_{2}\right\}$. Then, by (1.70),(1.78),(1.69) and (1.79), $3 \varphi=$ $d_{G}(s)=d_{G}\left(s, z_{1}\right)+d_{G}\left(s, z_{2}\right)+d_{G}\left(s, M_{1} \cap M_{2}\right)=h_{G}^{r}\left(z_{1}\right)-h_{G-s}^{r-\varphi}\left(z_{1}\right)+\varphi+$ $h_{G}^{r}\left(z_{2}\right)-h_{G-s}^{r-\varphi}\left(z_{2}\right)+\varphi+d_{G}\left(s, M_{1} \cap M_{2}\right) \leq 2 \varphi+2 \leq 3 \varphi$. Thus $h_{G-s}^{r-\varphi}\left(z_{1}\right)=0$, so $z_{1} \in \mathcal{C}$, that is, (1.75) is violated for $T$, contradiction.

Case 2 If $M_{1}, M_{2}, M_{3} \in \mathcal{M}$. Then, by (1.80), (1.78), (1.79), $1 \leq d_{G}\left(M_{3}-\right.$ $\left.z_{3}, z_{3}\right)=d_{G}\left(M_{1} \cap M_{2}, z_{3}\right) \leq \bar{d}_{G}\left(M_{1}, M_{2}\right)-d_{G}\left(M_{1} \cap M_{2}, s\right) \leq 2-2=0$, contradiction. The proof of the claim is finished.

Since $T$ satisfies (1.75), so does $T^{\prime}$ and the proof of Lemma 3 is complete.

Let $G^{\prime}$ be the $T$-split of $G$ from Lemma 3. Let us denote the new vertex of $G^{\prime}$ of degree $|T|$ by $t$. Wlog. $d_{1} \geq d_{2} \geq \ldots \geq d_{p}$. If $d_{p}=|T|$ then let $f^{\prime}(s):=$ $\left(d_{1}, \ldots, d_{p-1}\right)$ otherwise $\left(|T|=2, d_{1} \geq 4\right)$ let $f^{\prime}(s):=\left(d_{1}-2, d_{2}, \ldots, d_{p}\right)$. Then $\left(G^{\prime}, f^{\prime}(s)\right)$ satisfies (1.72) and $(1.73)$ and $z\left(G^{\prime}\right)<z(G)$, so by induction, $G^{\prime}$ has an r-edge-connected $f^{\prime}(s)$-detachment $G^{\prime \prime}$. Then, in the former case $G^{\prime \prime}$, in the latter case the graph obtained from $G^{\prime \prime}$ by identifying $s_{1}$ and $t$, is an $\mathbf{r}$-edge-connected $f(s)$-detachment of $G$.

\subsubsection{A short proof of Theorem 15}

I hope that the reader will find interesting the following shortened proof from [35]. I ask the reader not to be frightened by the technical aspects of the proof and to make figures to help to understand the proofs. In this section we call a set $X \subset V$ tight (resp. dangerous) if $d(X)=k$ (resp. $d(X) \leq k+1)$. We will abbreviate $k$-admissible by admissible. In order to have a more convenient notation, $e \in P_{j}$ will also be denoted by $c(e)=j$. 


\section{Preliminaries}

The following easy observations are from [16].

Proposition 1. (a) $\{s u, s v\}$ is admissible if and only if there is no dangerous set containing $u$ and $v$.

(b) For any edge su, there exist at most two dangerous sets $M_{1}$ and $M_{2}$ so that $u \in M_{1} \cap M_{2}$ and $\{v:\{s u, s v\}$ is not admissible $\} \subseteq M_{1} \cup M_{2}$.

(c) For a tight set $T,\{s u, s v\}$ is admissible in $G$ if and only if it is admissible in $G / T$.

The following proposition contains some technical remarks.

Proposition 2. (a) $d(X)-k \geq 2 d(s, X)-d(s) \forall X \subset V$ where equality holds if and only if $d(V-X)=k$.

(b) If $k \geq 3$ and $d(X) \leq k+2$ then $G[X]$ is connected.

(c) If $k$ is odd, $X_{1}, X_{2}, X_{3}$ are disjoint tight sets, $d\left(\cup_{i=1}^{3} X_{i}\right)=k+2$ and $d\left(X_{1}, X_{3}\right)=0$, then $d\left(X_{1}, X_{2}\right)=d\left(X_{2}, X_{3}\right)=\frac{k-1}{2}$.

Proof. (a) By (1.34), $d(X)-k=d(V-X)-k+d(s, X)-(d(s)-d(s, X)) \geq$ $2 d(s, X)-d(s)$.

(b) For a set $\emptyset \neq Y \subset X$, by (1.2) and (1.34), $(k+2)+2 d(Y, X-Y) \geq$ $d(X)+2 d(Y, X-Y)=d(Y)+d(X-Y) \geq k+k \geq k+3$, and (b) follows.

(c) By (1.2) and (1.34), $\forall i \in\{1,3\}, 2 k=d\left(X_{2}\right)+d\left(X_{i}\right)=d\left(X_{2} \cup X_{i}\right)+$ $2 d\left(X_{2}, X_{i}\right) \geq k+2 d\left(X_{2}, X_{i}\right)$, thus, by parity, $2 d\left(X_{2}, X_{i}\right) \leq k-1$. Then $3 k=\sum_{i=1}^{3} d\left(X_{i}\right)=d\left(\cup_{i=1}^{3} X_{i}\right)+\sum_{i \neq j} 2 d\left(X_{i}, X_{j}\right) \leq(k+2)+2(k-1)+0=3 k$, and (c) follows.

We present now some important properties of $C_{4^{-}}$and $C_{6}$-obstacles.

Proposition 3. (a) If $\mathcal{A}$ is a $C_{4}$-obstacle, then $d\left(s, A_{i}\right) \geq 1 \forall A_{i} \in \mathcal{A}$.

(b) If $\left\{A_{1}, A_{2}, A_{3}, A_{4}\right\}$ is a $C_{4}$-obstacle, then for each set $\emptyset \neq X \subseteq A_{i}$, $d(V-X) \geq k+2$ with equality only if $d(X)=k$.

(c) If $\left\{A_{1}, a_{2}, a_{3}, a_{4}\right\}$ is a $C_{4}$-obstacle, then for each set $X$ with $X \cap A_{1} \neq \emptyset$ and $a_{3} \in X, d(X) \geq k+2$.

(d) If $\left\{A_{1}, \ldots, A_{6}\right\}$ is a $C_{6}$-obstacle, then for every allowed pair $\{s x, s y\}$, $G_{x, y}$ contains a $C_{4}$-obstacle.

(e) If $\left\{A_{1}, a_{2}, \ldots, a_{6}\right\}$ is a $C_{6}$-obstacle and, for a set $X \neq V, X \cap A_{1} \neq \emptyset$, $y \in X \cap\left(a_{3} \cup a_{5}\right)$ and $d(X) \leq k+2$, then (e1) $d(X)=k+2$ and (e2) $X \cup A_{1}$ is the union of three consecutive sets in $\mathcal{A}$.

Proof. (a) Suppose wlog. $d\left(s, A_{1}\right)=0$. Then, by (1.27) and (1.26), $d\left(A_{1}, A_{2}\right)+$ $d\left(A_{1}, A_{4}\right)=d\left(A_{1}\right)=k$, so, since $k$ is odd, wlog. $d\left(A_{1}, A_{2}\right) \geq \frac{k+1}{2}$. Then, by (1.34), (1.2) and (1.26), $k \leq d\left(A_{1} \cup A_{2}\right)=d\left(A_{1}\right)+d\left(A_{2}\right)-2 d\left(A_{1}, A_{2}\right) \leq$ $k+k-(k+1)=k-1$, contradiction.

(b) Let $j:=i+2$ if $i \leq 2$ and $j:=i-2$ if $i \geq 3$. Then, by (1.34), Proposition $3(\mathrm{a}),(1.28)$ and $(1.29), k+2 \leq d(X)+2 d\left(s, A_{j}\right)=d(X)-\left(\frac{d(s)}{2}-\right.$ $\left.d\left(s, A_{j}\right)\right)+\frac{d(s)}{2}+d\left(s, A_{j}\right)=d(V-X)$ and (b) follows. 
(c) If $a_{2}, a_{4} \notin X$, then, by $X \cap A_{1} \neq \emptyset,(1.34),(1.26)$ and $(1.27), d(X)=$ $d\left(X \cap A_{1}\right)+d\left(a_{3}\right)=k+k \geq k+2$. If $a_{2}, a_{4} \in X$, then, by Proposition 3(b), $d(X) \geq k+2$. Otherwise, wlog. $a_{2} \notin X$ and $a_{4} \in X$. By Proposition 3(b), $d\left(X \cup A_{1}\right) \geq k+2$. Then, by (1.2) and (1.34), $d(X) \geq d\left(X \cap A_{1}\right)+d(X \cup$ $\left.A_{1}\right)-d\left(A_{1}\right) \geq k+(k+2)-k=k+2$.

(d) Wlog. $x \in A_{1}$. By (1.2), (1.30), (1.31), $d\left(A_{i} \cup A_{i+1}\right)=d\left(A_{i}\right)+d\left(A_{i+1}\right)-$ $2 d\left(A_{i}, A_{i+1}\right)=k+k-(k-1)=k+1$. Then, since $\{s x, s y\}$ is admissible, $y \notin A_{2} \cup A_{6}$ by Proposition 1(a). $\{s x, s y\}$ is allowed so, by (1.33), $y \notin A_{4}$. Thus wlog. $y \in A_{3}$. Then $\left\{A_{1} \cup A_{2} \cup A_{3}, A_{4}, A_{5}, A_{6}\right\}$ is a $C_{4}$-obstacle in $G_{x, y}$.

(e) Let $X^{*}:=X \cup A_{1}$. By $(1.31), d_{G-s}\left(X^{*}\right) \geq k-1$ where equality holds if and only if $X^{*}$ is the union of $2<l<6$ consecutive sets in $\mathcal{A}$. By Proposition $2(\mathrm{a}), d(s, X) \leq 4$, by $(1.32), d\left(s, A_{1}\right)=1$ and $d(s, V)=6$ so $X^{*} \neq V$. By (1.30), $d\left(A_{1}\right)=k$, by $X \cap A_{1} \neq \emptyset$ and (1.34), $d\left(X \cap A_{1}\right) \geq k$, so by $(1.2),(k+2)+k \geq d(X)+d\left(A_{1}\right) \geq d\left(X \cap A_{1}\right)+d\left(X^{*}\right) \geq k+d\left(X^{*}\right)$, so $k+2 \geq d\left(X^{*}\right)$ and if equality holds then $d(X)=k+2$. Then, by Proposition 2(b), $G\left[X^{*}\right]$ is connected. Since $d_{G-s}\left(y, A_{1}\right)=0, X^{\prime}:=X-\left(y \cup A_{1}\right) \neq \emptyset$. Then $k+2 \geq d\left(X^{*}\right)=d\left(s, X^{*}\right)+d_{G-s}\left(X^{*}\right) \geq d(s, y)+d\left(s, X^{\prime}\right)+d\left(s, A_{1}\right)+$ $d_{G-s}\left(X^{*}\right) \geq 1+1+1+(k-1)$, so $d\left(X^{*}\right)=k+2$ and hence $d(X)=k+2$ (implying (e1)), $d\left(s, X^{\prime}\right)=1$ and $d_{G-s}\left(X^{*}\right)=k-1$, thus (e2) is satisfied.

The following lemma will allow us to easily find an allowed pair. The main difficulty of the proof of Theorem 15 will be to show that there exists an allowed pair whose splitting off creates no $C_{4^{-}}$and no $C_{6}$-obstacle.

Lemma 4. If $G$ contains no $C_{4}$-obstacle and (1.37) is satisfied then each edge su belongs to an allowed pair.

Proof. Let $S:=\{s v \in E:\{s u, s v\}$ is admissible $\}$. Suppose $s u$ belongs to no allowed pair. Then every $s v \in S$ and $s u$ belong to the same $P_{j}$. Then, by (1.37), $\frac{d(s)}{2} \geq\left|P_{j}\right| \geq|S|+1$, so $|S| \leq \frac{d(s)}{2}-1$ and if equality holds then $\frac{d(s)}{2}=\left|P_{j}\right|$. It also follows, by Proposition 1(b), that there are at most two dangerous sets $M_{1}$ and $M_{2}$ so that $u \in M_{1} \cap M_{2}$ and $\left\{v_{i}: s v_{i} \in \delta(s)-S\right\} \subseteq M_{1} \cup M_{2}$. In fact there are exactly two, because, by Proposition 2, $d\left(M_{1} \cup M_{2}\right)-k \geq$ $2 d\left(s, M_{1} \cup M_{2}\right)-d(s)=2(d(s)-|S|)-d(s) \geq d(s)-2\left(\frac{d(s)}{2}-1\right)=2$, and if equality holds then $d\left(V-M_{1} \cup M_{2}\right)=k$ and $|S|=\frac{d(s)}{2}-1$. The following claim provides a contradiction.

Claim. $\left\{A_{1}=M_{1} \cap M_{2}, A_{2}=M_{1}-M_{2}, A_{3}=V-M_{1} \cup M_{2}, A_{4}=M_{2}-M_{1}\right\}$ forms a $C_{4}$-obstacle.

Proof. Note that $A_{i} \neq \emptyset 1 \leq i \leq 4$ and $\cup_{i=1}^{4} A_{i}=V$. By (1.2), (1.34) and $d\left(M_{1} \cup M_{2}\right) \geq k+2,2(k+1) \geq d\left(M_{1}\right)+d\left(M_{2}\right)=d\left(A_{1}\right)+d\left(M_{1} \cup M_{2}\right)+$ $2 d\left(M_{1}, M_{2}\right) \geq k+(k+2)$, so $d\left(A_{1}\right)=k, d\left(M_{1} \cup M_{2}\right)=k+2$ and hence $d\left(A_{3}\right)=$ $k$ and $\frac{d(s)}{2}=\left|P_{j}\right|$ so $(1.28)$ is satisfied, and $d\left(A_{2}, A_{4}\right)=d\left(M_{1}, M_{2}\right)=0$. By (1.3) and (1.34), 2(k+1) $\geq d\left(M_{1}\right)+d\left(M_{2}\right)=d\left(A_{2}\right)+d\left(A_{4}\right)+2 d\left(A_{1}, A_{3}+s\right) \geq$ $k+k+2 d\left(A_{1}, A_{3}\right)+2 d\left(A_{1}, s\right) \geq 2 k+0+2$, so $d\left(A_{2}\right)=d\left(A_{4}\right)=k, d\left(A_{1}, A_{3}\right)=0$ 
and $d\left(s, A_{1}\right)=1$. It also follows that $\delta\left(A_{1} \cup A_{3}\right) \cap \delta(s)=P_{j}$, so (1.26), (1.27) and (1.29) are satisfied. This completes the proof of the claim and also of Lemma 4.

\section{Proof of the necessity of Theorem 15}

Suppose there exists a graph that has a complete allowed splitting off $\left\{\left\{e_{i}, f_{i}\right\}: 1 \leq i \leq \frac{d_{G}(s)}{2}\right\}$ and violates (1.37) or (1.36). Choose such a graph $G$ with $d_{G}(s)$ minimum. For every $1 \leq i \leq \frac{d_{G}(s)}{2}, 1 \leq j \leq r,\left|P_{j} \cap\left\{e_{i}, f_{i}\right\}\right| \leq 1$ so (1.37) is satisfied, whence $G$ contains a $C_{4^{-}}$or a $C_{6}$-obstacle. By Proposition $3(\mathrm{a})$ and $(1.32), d_{G}(s) \neq 0$. Then, either by (1.28) and (1.29) or by Proposition $3(\mathrm{~d}), G_{e_{1}, f_{1}}$ contains a $C_{4}$-obstacle, $G_{e_{1}, f_{1}}$ has of course a complete allowed splitting off, and $d_{G_{e_{1}, f_{1}}}(s)<d_{G}(s)$, contradiction.

\section{Proof of the sufficiency of Theorem 15}

Induction on $|V|$. By Proposition 1(c), we may assume that

every tight set is a singleton.

Wlog. $\left|P_{1}\right|$ is maximum. By Lemma 4 , there is an allowed pair $\{e=s x, f=s y\}$ with $s x \in P_{1}$. If, after splitting off this pair, we get stuck by a $C_{6}$-obstacle, then we can get rid of it by the following lemma.

Lemma 5. Suppose that $G^{\prime}:=G_{e, f}$ contains a $C_{6}$-obstacle $\mathcal{A}=\left\{A_{1}, \ldots, A_{6}\right\}$. Then there exists an edge $f^{\prime}=s y^{\prime}$ such that $\left\{e, f^{\prime}\right\}$ is allowed in $G$ and $G^{\prime \prime}:=G_{e, f^{\prime}}$ satisfies (1.34), (1.37) and (1.36).

Proof. The suitable $y^{\prime}$ will be chosen as follows: Wlog. $x \in A_{1}$. Then, by (1.30) and (1.81), $A_{j}=a_{j} \forall 2 \leq j \leq 6$. By (1.33), $c\left(s a_{3}\right) \neq c\left(s a_{5}\right)$ so either $c\left(s a_{3}\right) \neq 1$ in which case let $y^{\prime}:=a_{3}$ or $c\left(s a_{5}\right) \neq 1$ and then let $y^{\prime}:=a_{5}$. We show that $y^{\prime}$ will do. For each set $X$ with $x, y^{\prime} \in X \neq V$, by Proposition $3(\mathrm{e}), k+2 \leq d_{G^{\prime}}(X) \leq d_{G}(X)$, so by Proposition 1(a), $\left\{e, f^{\prime}\right\}$ is admissible in $G$, thus $G^{\prime \prime}$ satisfies (1.34). Since $c(s x)=1 \neq c\left(s y^{\prime}\right), G^{\prime \prime}$ satisfies (1.37) and $\left\{e, f^{\prime}\right\}$ is allowed in $G$. It remains to show that $G^{\prime \prime}$ satisfies (1.36). Since $x y \in E\left(G^{\prime}\right)$, either Case a: $y \in A_{1}$ or Case b: wlog. $y \in A_{2}$. In both cases we suppose indirect that $G^{\prime \prime}$ contains a $C_{4^{-}}$(Case 1 ) or a $C_{6^{-}}$-obstacle (Case 2) $\mathcal{A}^{\prime}$.

Case a: Wlog. $y^{\prime}=a_{5}$ and $x \in A_{1}^{\prime}$. Suppose $y^{\prime} \notin A_{1}^{\prime}$. By (1.30), $k+2=$ $d_{G^{\prime}}\left(A_{1}\right)+2=d_{G}\left(A_{1}\right)$. By (1.26) or (1.30), $d_{G}\left(A_{1}^{\prime}\right)=k$ and then, by (1.81), $\left|A_{i}^{\prime}\right|=1 \forall A_{i}^{\prime} \in \mathcal{A}^{\prime}$. Hence $|V|=4$ or 6 . But $|V| \geq 6$ because $G^{\prime}$ contains the $C_{6}$-obstacle $\mathcal{A}$, so $|V|=6$. Thus $A_{1}^{\prime}=A_{1}$ and hence $k=d_{G^{\prime \prime}}\left(A_{1}^{\prime}\right)=$ $d_{G^{\prime \prime}}\left(A_{1}\right)=d_{G}\left(A_{1}\right)$, contradiction. Thus $y^{\prime} \in A_{1}^{\prime}$ and $d_{G}\left(A_{1}^{\prime}\right)=k+2$. Since $d_{G^{\prime}}\left(A_{1}^{\prime}\right) \leq d_{G}\left(A_{1}^{\prime}\right), A_{1}^{\prime} \cup A_{1}$ is the union of three consecutive sets in $\mathcal{A}$ by Proposition 3(e). 
Case a1: Then $3=\left|V-\left(A_{1} \cup A_{1}^{\prime}\right)\right| \leq\left|V-A_{1}^{\prime}\right|=3$ by (1.81) so $A_{1} \subset A_{1}^{\prime}$ thus, by (1.31), wlog. $A_{j}^{\prime}=a_{j} 2 \leq j \leq 4$. By (1.33) for $\mathcal{A}$, there is a $w \in A_{1}$ with $c(s w)=c\left(s a_{4}\right)$ but $w \in A_{1}^{\prime}$ and $a_{4} \in A_{4}^{\prime}$, contradiction by (1.29) for $\mathcal{A}^{\prime}$.

Case a2: Then $a_{6} \in A_{1}^{\prime}$. Wlog. $A_{2}^{\prime}=a_{4}$ and $A_{3}^{\prime}=a_{3}$. Then, by (1.33) for $\mathcal{A}$ and $\mathcal{A}^{\prime}, c\left(s a_{3}\right)=c\left(s a_{6}\right) \neq c\left(s a_{3}\right)$, contradiction, and we are done in Case a.

Case b: Then, by (1.30) and (1.81), $A_{1}=a_{1}$ so $|V|=6$.

Case b1: Since $d_{G^{\prime \prime}}\left(s, y^{\prime}\right)=0$, wlog. $x, y^{\prime} \in A_{1}^{\prime}$ and $d_{G}\left(A_{1}^{\prime}\right)=k+2$. Since $d_{G^{\prime}}\left(A_{1}^{\prime}\right) \leq d_{G}\left(A_{1}^{\prime}\right), d_{G^{\prime}}\left(A_{1}^{\prime}\right)=k+2$ and $A_{1}^{\prime} \cup A_{1}$ is the union of three consecutive sets in $\mathcal{A}$ by Proposition $3(\mathrm{e})$. Then $d_{G^{\prime}}\left(A_{1}^{\prime}\right)=d_{G}\left(A_{1}^{\prime}\right)$ so $y^{\prime}=a_{5}$. Thus $A_{1}^{\prime}=\left\{a_{5}, a_{6}, a_{1}\right\}$. Wlog. $A_{j}^{\prime}=a_{j} 2 \leq j \leq 4$ by (1.31) for $\mathcal{A}$ and (1.27) for $\mathcal{A}^{\prime}$. By (1.33) for $\mathcal{A}, c\left(s a_{1}\right)=c\left(s a_{4}\right)$, contradiction by (1.29) for $\mathcal{A}^{\prime}$.

Case b2: Note that, by $(1.32), d_{G}\left(s, a_{1}\right)=d_{G}\left(s, a_{2}\right)=2$ and $d_{G}\left(s, a_{h}\right)=$ $1(3 \leq h \leq 6)$. Then $d_{G^{\prime \prime}}\left(s, a_{2}\right)=2$ that is, by $(1.32)$, a contradiction, and we are done in Case b, and the proof of Lemma 5 is complete.

The following lemma allows us to get rid of a $C_{4}$-obstacle if during the splitting off we get stuck with one.

Lemma 6. Suppose that $G^{\prime}:=G_{e, f}$ contains a $C_{4}$-obstacle $\mathcal{A}:=\left\{A_{1}, A_{2}, A_{3}\right.$, $\left.A_{4}\right\}$. Then there exists an allowed pair $e^{\prime}=s x^{\prime}, f^{\prime}=s y^{\prime}$ in $G$ such that $G^{\prime \prime}:=G_{e^{\prime}, f^{\prime}}$ satisfies (1.34), (1.37) and (1.36).

Proof. Wlog. $x \in A_{1}$. Since $x y \in E\left(G^{\prime}\right)$, either Case a: wlog. $y \in A_{2}$ or Case b: $y \in A_{1}$. By (1.26) and (1.81), $A_{j}=a_{j} \forall 2 \leq j \leq 4$, in Case a: $A_{1}=a_{1}$ so $|V|=4$ and in Case b: $d_{G}\left(A_{1}\right)=k+2$.

Case a: We chose $e^{\prime}$ and $f^{\prime}$ as follows. If there exists an edge $g=s a_{3}$ with $c(g) \neq c(e)$ then let $e^{\prime}:=e, f^{\prime}:=g$. Otherwise, since $\mathcal{A}$ is not a $C_{4}$-obstacle in $G$, there is an edge $h=s a_{1}$ with $c(h) \neq c(e)$ and then let $e^{\prime}:=s a_{3}, f^{\prime}:=h$. We show that this pair will do. Note that $\left\{x^{\prime}, y^{\prime}\right\}=\left\{a_{1}, a_{3}\right\}$. For each set $X$ with $x^{\prime}, y^{\prime} \in X \neq V$, either $X=\left\{x^{\prime}, y^{\prime}\right\}$ and then, by (1.26) and (1.27), $d_{G}(X)=$ $d_{G}\left(a_{1}\right)+d_{G}\left(a_{3}\right)=k+k \geq k+2$ or, by $X \neq V, \exists i \in\{2,4\} X=\left\{a_{1}, a_{3}, a_{i}\right\}$ and then, by Proposition $3(\mathrm{~b}), k+2 \leq d_{G^{\prime}}(X) \leq d_{G}(X)$. In both cases, by Proposition 1(a), $\left\{e^{\prime}, f^{\prime}\right\}$ is admissible in $G$, thus $G^{\prime \prime}$ satisfies (1.34). Since $c\left(e^{\prime}\right)=c(e), G^{\prime \prime}$ satisfies (1.37) and $\left\{e^{\prime}, f^{\prime}\right\}$ is allowed in $G$. It remains to show that $G^{\prime \prime}$ satisfies (1.36). Suppose not. Then, since $|V|=4, G^{\prime \prime}$ contains a $C_{4^{-}}$ obstacle $\mathcal{A}^{\prime}:=\left\{A_{1}^{\prime}, A_{2}^{\prime}, A_{3}^{\prime}, A_{4}^{\prime}\right\}$. Since $a_{1} a_{3} \in E\left(G^{\prime \prime}\right)$, wlog. $A_{1}^{\prime} \cup A_{2}^{\prime}=a_{1} \cup a_{3}$ and $A_{3}^{\prime} \cup A_{4}^{\prime}=a_{2} \cup a_{4}$. By Proposition $3(\mathrm{a}), d_{G^{\prime \prime}}\left(s, A_{i}^{\prime}\right) \geq 1$ so $d_{G}\left(s, A_{i}^{\prime}\right) \geq 2 i \in$ $\{1,2\}$. By (1.28) and (1.29) for $\mathcal{A}$ in $G^{\prime}$, there exist $1 \leq l \leq r$ and $j \in\{1,2\}$ such that for every edge $s d \in E\left(G^{\prime}\right)$ with $d \in A_{j} \cup A_{j+2}, c(s d)=l$. Then there exist $s d_{1}, s d_{2} \in E\left(G^{\prime \prime}\right)$ with $d_{1} \in A_{j}, d_{2} \in A_{j+2}$ and $c\left(s d_{1}\right)=c\left(s d_{2}\right)=l$. This contradicts (1.29) for $\mathcal{A}^{\prime}$.

Case b: Either we reduce this case to a case already seen or we show that the following edge pair $e^{\prime}, f^{\prime}$ will do. Let $g:=s a_{3}$. If $c(g) \neq c(e)$, then let $e^{\prime}:=e$ and $f^{\prime}:=g$, otherwise, let $e^{\prime}:=g$ and $f^{\prime}:=f$. Since $c\left(e^{\prime}\right)=c(e)$, 
$G^{\prime \prime}$ satisfies (1.37). By Propositions 1(a) and 3(c), $\left\{e^{\prime}, f^{\prime}\right\}$ is admissible in $G$, so allowed in $G$ and (1.34) is satisfied. If $G^{\prime \prime}$ satisfies (1.36), then we are done. Otherwise, either $G^{\prime \prime}$ contains a $C_{6}$-obstacle, and then, by Lemma 5 , we are done, or $G^{\prime \prime}$ contains a $C_{4}$-obstacle $\mathcal{A}^{\prime}:=\left\{A_{1}^{\prime}, A_{2}^{\prime}, A_{3}^{\prime}, A_{4}^{\prime}\right\}$. Wlog. $x^{\prime}, y^{\prime} \in A_{1}^{\prime}$, otherwise restarting the proof by $e^{\prime}$ and $f^{\prime}$ we are in Case a. Then, by (1.26) and (1.81), $\left|A_{j}^{\prime}\right|=1 \forall 2 \leq j \leq 4$ and $d_{G}\left(A_{1}^{\prime}\right)=k+2$. By Proposition 2(b), $G\left[A_{1}^{\prime}\right]$ is connected, so, by (1.27), wlog. $V-a_{4} \subseteq A_{1}^{\prime} \cup A_{1}$. First suppose that $A_{1}^{\prime} \cup A_{1}=V$. Then, by Propositions 3(b) applied for $G^{\prime}$, $d_{G^{\prime}}\left(A_{1}^{\prime}\right)=k+2$ and $k=d_{G^{\prime}}\left(V-A_{1}^{\prime}\right)$. The common edge of $\{e, f\}$ and $\left\{e^{\prime}, f^{\prime}\right\}$ enters $A_{1} \cap A_{1}^{\prime}$, so $d_{G^{\prime}}\left(V-A_{1}^{\prime}\right)=d_{G}\left(V-A_{1}^{\prime}\right)$ thus, by $(1.81), 1=\left|V-A_{1}^{\prime}\right|(=3)$, contradiction, so $A_{1}^{\prime} \cup A_{1}=V-a_{4}$. Then, by Proposition 3(b) again applied for $G^{\prime}, d_{G^{\prime}}\left(A_{1}^{\prime} \cup A_{1}\right) \geq k+2$. Then, by (1.2) and (1.34), $d_{G}\left(A_{1}^{\prime} \cap A_{1}\right)=k$ thus, by (1.81), $\left|A_{1}^{\prime} \cap A_{1}\right|=1$, say $A_{1}^{\prime} \cap A_{1}=a_{1}$. Then it follows that $|V|=6$, say $V=\left\{a_{1}, a_{2}, \ldots, a_{6}\right\}$. Note that $d_{G}\left(a_{i}\right)=k 1 \leq i \leq 6$. By Proposition 3(a) for $\mathcal{A}$ and for $\mathcal{A}^{\prime}, 1 \leq d_{G}\left(s a_{i}\right) 1 \leq i \leq 6$ so $6 \leq d_{G}(s)$. The following claim provides a contradiction.

Claim. $\left\{a_{1}, a_{2}, \ldots, a_{6}\right\}$ forms a $C_{6}$-obstacle in $G$.

Proof. We start with some structural observations.

Proposition 4. (a) $A_{1}=\left\{a_{1}, a_{5}, a_{6}\right\}, A_{i}=a_{i} 2 \leq i \leq 4, A_{1}^{\prime}=\left\{a_{1}, a_{2}, a_{3}\right\}$, wlog. $A_{2}^{\prime}=a_{6}, A_{3}^{\prime}=a_{5}, A_{4}^{\prime}=a_{4}$, (b) $d_{G}\left(a_{1}, a_{2}\right)=d_{G}\left(a_{2}, a_{3}\right)=d_{G}\left(a_{1}, a_{6}\right)=$ $d_{G}\left(a_{5}, a_{6}\right)=\frac{k-1}{2}$, (c) $\{x, y\}=\left\{a_{1}, a_{5}\right\}$.

Proof. We know that $A_{1}^{\prime}=\left\{a_{1}, a_{2}, a_{3}\right\}$ and $A_{1}=\left\{a_{1}, a_{5}, a_{6}\right\}$. Then, by (1.27) for $\mathcal{A}, d_{G}\left(a_{1}, a_{3}\right)=0$ so, by Proposition $2(\mathrm{c}), d_{G}\left(a_{1}, a_{2}\right)=d_{G}\left(a_{2}, a_{3}\right)=\frac{k-1}{2}$. Wlog. $A_{2}^{\prime}=a_{6}$. Suppose that $A_{4}^{\prime}=a_{5}$. Then, by (1.27) for $\mathcal{A}^{\prime}, d_{G}\left(a_{5}, a_{6}\right)=0$ so, by Proposition 2(c), $d_{G}\left(a_{1}, a_{5}\right)=d_{G}\left(a_{1}, a_{6}\right)=\frac{k-1}{2}$. Then $k=d_{G}\left(a_{1}\right) \geq$ $d_{G}\left(a_{1}, a_{2}\right)+d_{G}\left(a_{1}, a_{5}\right)+d_{G}\left(a_{1}, a_{6}\right)+d_{G}\left(a_{1}, s\right) \geq 3 \frac{k-1}{2}+1$, that is $k \leq 1$, contradiction. Thus $A_{4}^{\prime}=a_{4}$ and $A_{3}^{\prime}=a_{5}$, that is, (a) is satisfied. Then, by (1.27) for $\mathcal{A}^{\prime}, d_{G}\left(a_{1}, a_{5}\right)=0$ so, by Proposition $2(\mathrm{c}), d_{G}\left(a_{1}, a_{6}\right)=d_{G}\left(a_{5}, a_{6}\right)=\frac{k-1}{2}$ and (b) is satisfied. By definition, $\{x, y\} \cap\left\{x^{\prime}, y^{\prime}\right\}=a_{1}$, so $a_{1} \in\{x, y\}$. Suppose $a_{5} \notin\{x, y\}$. Then $\{x, y\}=\left\{a_{1}, a_{6}\right\}$. By (1.2), (1.30) and (b), $d_{G}\left(\left\{a_{1}, a_{6}\right\}\right)=d_{G}\left(a_{1}\right)+d_{G}\left(a_{6}\right)-2 d_{G}\left(a_{1}, a_{6}\right)=k+k-(k-1)=k+1$, hence, by Proposition 1(a), $\{s x, s y\}$ is not admissible in $G$, contradiction, thus (c) is satisfied.

By (1.29) for $\mathcal{A}^{\prime}, c\left(s a_{2}\right) \neq c\left(s a_{4}\right)$ so $\delta_{G^{\prime}}\left(A_{1} \cup A_{3}\right) \cap \delta_{G^{\prime}}(s)=P_{l}^{\prime}$ in (1.29) for $\mathcal{A}$ for some $l$ with $\left|P_{l}\right| \geq\left|P_{l}^{\prime}\right|=\frac{d_{G^{\prime}}(s)}{2}=\frac{d_{G}(s)}{2}-1$. By (1.29) for $\mathcal{A}$, $c\left(s a_{6}\right) \neq c\left(s a_{4}\right)$ so $\delta_{G^{\prime \prime}}\left(A_{1}^{\prime} \cup A_{3}^{\prime}\right) \cap \delta_{G^{\prime \prime}}(s)=P_{l^{\prime}}$ in (1.29) for $\mathcal{A}^{\prime}$ for some $l^{\prime}$ with $\left|P_{l^{\prime}}\right| \geq\left|P_{l^{\prime}}^{\prime}\right|=\frac{d_{G^{\prime \prime}}(s)}{2}=\frac{d_{G}(s)}{2}-1$. In particular, $c\left(s a_{2}\right)=c\left(s a_{5}\right)=l^{\prime}$. By (1.29) for $\mathcal{A}, l=c\left(s a_{3}\right) \neq c\left(s a_{2}\right)=l^{\prime}$ thus, by Proposition 4(c), $e=e^{\prime}=$ $s a_{1}, f=s a_{5}, f^{\prime}=s a_{3}$. Since $\{e, f\}$ and $\left\{e^{\prime}, f^{\prime}\right\}$ are allowed, $l \neq 1 \neq l^{\prime}$. Then, by the maximality of $P_{1},\left|P_{1}\right| \geq\left|P_{l}\right| \geq \frac{d_{G}(s)}{2}-1 . d_{G}(s) \geq\left|P_{1}\right|+\left|P_{l}\right|+\left|P_{l^{\prime}}\right| \geq$ $3\left(\frac{d_{G}(s)}{2}-1\right)$, that is, $d_{G}(s) \leq 6$. Then $d_{G}(s)=6$ and $\left|P_{1}\right|=\left|P_{l}\right|=\left|P_{l^{\prime}}\right|=2$, 
namely $P_{1}=\left\{s a_{1}, s a_{4}\right\}, P_{l}=\left\{s a_{3}, s a_{6}\right\}, P_{l^{\prime}}=\left\{s a_{2}, s a_{5}\right\}$, so (1.32) and (1.33) are satisfied. We have already seen that (1.30) is satisfied. By (1.26) and (1.27) for $\mathcal{A}^{\prime}$ and for $\mathcal{A}$, Proposition 4(b) and (1.32), $d_{G}\left(a_{5}, a_{4}\right)=\frac{k-1}{2}=d_{G}\left(a_{3}, a_{4}\right)$. Then, by Proposition 4(b), (1.31) is satisfied. This completes the proof of the claim and also of Lemma 6.

By Lemmas 4, there exists an allowed pair, and then, by Lemmas 5 and 6 , there exists one that does not create a $C_{4^{-}}$or $C_{6}$-obstacle, so by induction, there exists a complete allowed splitting off and Theorem 15 is proved.

Acknowledgement. I thank T. Király for the fruitful discussions on this topic.

\section{References}

1. J. Bang-Jensen, A. Frank, B. Jackson, Preserving and increasing local edgeconnectivity in mixed graphs, SIAM J. Discrete Math. Vol. 8 No. 2 (1995) $155-178$

2. J. Bang-Jensen, H. Gabow, T. Jordán, Z. Szigeti, Edge-connectivity augmentation with partition constraints, SIAM J. Discrete Math. Vol. 12 No. 2 (1999) 160-207.

3. J. Bang-Jensen, B. Jackson, Augmenting hypergraphs by edges of size two, Math. Program. Vol. 84 No. 3 (1999) 467-481.

4. J. Bang-Jensen, T. Jordán, Edge-connectivity augmentation preserving simplicity, SIAM J. Discrete Math. Vol. 11 No. 4 (1998) 603-623.

5. J. Bang-Jensen, T. Jordán, Splitting off edges within a specified subset preserving the edge-connectivity of the graph, J. of Algorithms 37 (2000) 326-343.

6. A. Benczúr, A. Frank, Covering symmetric supermodular functions by graphs, Math. Program. Vol. 84 No. 3 (1999) 483-503.

7. A. Bernáth, T. Király, personal communication

8. G. R. Cai, Y. G. Sun, The minimum augmentation of any graph to $k$-edgeconnected graphs, Networks, 19 (1989) 151-172.

9. E. Cheng, T. Jordán, Successive edge-connectivity augmentation problems, Math. Program. Vol. 84 No. 3 (1999) 577-593.

10. B. Cosh, Vertex Splitting and Connectivity Augmentation in Hypergraphs, Ph.D. thesis, University of London (2000)

11. B. Cosh, B. Jackson, Z. Király, Local connectivity augmentation in hypergraphs is NP-complete, submitted

12. K. P. Eswaran, E. Tarjan, Augmentation problems, SIAM J. Comput., 5 (1976) 653-665.

13. T. Fleiner, T. Jordán, Covering and structure of crossing families, Math. Program. Vol. 84 No. 3 (1999) 505-518.

14. B. Fleiner, Detachments of vertices of graphs preserving edge-connectivity, SIAM J. Discrete Math. Vol. 18 No. 3 (2005) 581-591.

15. A. Frank, Augmenting graphs to meet edge-connectivity requirements, SIAM J. Discrete Math. Vol. 5 No. 1 (1992) 22-53.

16. A. Frank, On a theorem of Mader, Discrete Math. 101 (1992) 49-57. 
17. A. Frank, oral communication

18. R. Grappe, Z. Szigeti, Covering semi-monotone symmetric functions, Discrete Appl. Math. 156 (2008) 138-144.

19. T. Ishii, M. Hagiwara, Minimum augmentation of local edge-connectivity between vertices and vertex subsets in undirected graphs, Disc. Appl. Math. 154 (2006) 2307-2329.

20. T. Ishii, Minimum augmentation of edge-connectivity with monotone requirements in undirected graphs, Proc. of the 13th Australasian Symp. on Theory of Comp., (2007) 91-100.

21. B, Jackson, Some remarks on connectivity, vertex splitting and orientation in digraphs, J. Graph Theory, 12, (1988) 429-436.

22. T. Jordán, Two NP-Complete Augmentation Problems, IMADA, Odense Universitet, Preprints No. 8/1997.

23. T. Jordán, Constrained edge-splitting problems, SIAM J. Discrete Math. Vol. 17 No. 1 (2003) 88-102.

24. T. Jordán, Z. Szigeti, Detachments preserving local edge-connectivity of graphs, SIAM J. Discrete Math. Vol. 17 No. 1 (2003) 72-87.

25. T. Király, Merging hyperedges to meet edge-connectivity requirements, Egres Technical Reports, TR-2005-08 http://www.cs.elte.hu/egres/, 2004.

26. T. Király, Covering symmetric supermodular functions by uniform hyperedges, J. Combin. Theory Ser. B 91 (2004) 185-200.

27. Z. Király, oral communication

28. L. Lovász, Combinatorial problems and exercises, North-Holland, Amsterdam, (1979).

29. W. Mader, A reduction method for edge-connectivity in graphs, Ann. Discrete Math. 3 (1978) 145-164.

30. Z. Nutov, Approximating connectivity augmentation problems, In SODA 2005, 176-185.

31. Z. Szigeti, Hypergraph connectivity augmentation, Math. Program. Vol. 84 No. 3 (1999) 519-527.

32. Z. Szigeti, Edge-connectivity augmentation of graphs over symmetric parity families, to appear in Discrete Math.

33. Z. Szigeti, Edge-splittings preserving edge-connectivity of graphs, Discrete Applied Mathematics, 156 (2008) 1011-1018.

34. Z. Szigeti, A short proof on the local detachment theorem, Egres Technical Reports, TR-2004-09 http://www.cs.elte.hu/egres/, 2004.

35. Z. Szigeti, On partition constrained splitting off, Egres Technical Reports, TR2004-08 http://www.cs.elte.hu/egres/, 2004.

36. T. Watanabe, A. Nakamura, Edge-connectivity augmentation problems, Comp. System Sci. 35 (1987) 96-144. 